\title{
The role of the microRNA regulatory network in Alzheimer's disease: a bioinformatics analysis
}

\author{
Chenjing Sun ${ }^{1}$, Jianguo Liư ${ }^{1}$, Feng Duan ${ }^{1}$, Lin Cong ${ }^{2}$, Xiaokun Qi ${ }^{1}$
}

\begin{abstract}
${ }^{1}$ Department of Neurology, PLA Navy General Hospital, Haidian District, Beijing, China 2Department of Orthopedic Surgery, The First Hospital of China Medical University, Heping District, Shenyang City, Liaoning Province, China
\end{abstract}

Submitted: 21 September 2017; Accepted: 19 November 2017

Online publication: 18 March 2021

Arch Med Sci 2022; 18 (1): 206-222

DOI: https://doi.org/10.5114/aoms/80619

Copyright $\odot 2021$ Termedia \& Banach

\begin{abstract}
Introduction: Alzheimer's disease $(A D)$ is a neurodegenerative disease which presents with an earlier age of onset and increased symptom severity. The objective of this study was to evaluate the relationship between regulation of miRNAs and AD.

Material and methods: We completed a bioinformatic analysis of miRNA-AD studies through multiple databases such as TargetScan, Database for Annotation, Visualization and Integrated Discovery (DAVID), FunRich and String and assessed which miRNAs are commonly elevated or decreased in brain tissues, cerebrospinal fluid (CSF) and blood of AD patients. All identified articles were assessed using specific inclusion and exclusion criteria.

Results: MiRNAs related to AD of twenty-eight studies were assessed in this study. A wide range of miRNAs were up-regulated or down-regulated in tissues of AD patients' brain, blood and CSF. Twenty-seven differentially dysregulated miRNAs involved in amyloidogenesis, inflammation, tau phosphorylation, apoptosis, synaptogenesis, neurotrophism, neuron degradation, and activation of cell cycle entry were identified. Additionally, our bioinformatics analysis identified the top ten functions of common miRNAs in candidate studies. The functions of common up-regulated miRNAs primarily target the nucleus and common down-regulated miRNAs primarily target transcription, DNA-templated.

Conclusions: Comprehensive analysis of all miRNA studies reveals cooperation in miRNA signatures whether in brain tissues or in CSF and peripheral blood. More and more studies suggest that miRNAs may play crucial roles as diagnostic biomarkers and/or as new therapeutic targets in AD. According to biomarkers, we can identify the preclinical phase early, which provides an important time window for therapeutic intervention.
\end{abstract}

Key words: Alzheimer's disease, microRNAs, brain, cerebrospinal fluid, peripheral blood, Homo, bioinformatic analysis.

\section{Introduction}

Alzheimer's disease (AD) is a complex, age-related neurological disorder in the central nervous system (CNS), characterized by progressive memory loss, cognitive deficits and inability to perform activities of daily living that involves the progressive dysregulation of multiple biological pathways at multiple molecular, genetic, epigenetic, neurophysiological, and cognitive levels $[1,2]$. As yet there is no consensus regarding the presence of altered awareness of memory dysfunction in the preclinical and prodromal stages

\author{
Corresponding author: \\ Xiaokun Qi \\ Department of Neurology \\ PLA Navy General Hospital \\ 6 Fucheng Road \\ Haidian District \\ Beijing, 100048 \\ China \\ E-mail: bjqxk@sina.com
}


of the disease. Although AD is the most common senile dementia, it is often a dilemma to differentially diagnose the insidious and fatal disease from other equally heterogeneous neurodegenerative diseases such as Parkinson's disease, Lewy body dementia, frontal temporal dementia, vascular dementia, Creutzfeldt-Jakob disease, Gerstmann-Sträussler-Scheinker syndrome, and Huntington's disease. Neurofibrillary tangles (NFTs) in AD are principally composed of the hyperphosphorylated and aggregated microtubule-associated protein tau, which is one of the major AD hallmarks. In clinical practice, doctors often base diagnosis of $A D$ on clinical examinations, brain imaging and neuropsychological tests such as the Mini-Mental State Examination (MMSE). Also cerebrospinal fluid (CSF) biochemical assays of the hyperphosphorylated tau protein and the 42-amino-acid $A \beta$ peptide level can support the diagnosis. For diagnosing the disease in preclinical and prodromal stages and developing effective treatments, it is necessary to look for new biomedical approaches, concepts, and molecular targets. It is very useful to evaluate the role of miRNAs in $A D$ to find miRNA-based therapeutic drugs.

MicroRNAs (miRNAs) are a principal class of small RNA regulators binding the 3' UTRs of target mRNA and negatively post-transcriptionally regulating the expression of mRNA [3] and play key roles in multiple biological processes such as development, proliferation, metabolism, neural plasticity, inflammation, and apoptosis. Since the first reports of miRNA dysregulation in $A D$, it has been almost 10 years. There are three forms of altered miRNA abundance and speciation [4, 5]: 1 ) in brain tissues targeted by the AD process after post-mortem examination; 2) in blood serum, and 3 ) in cerebrospinal fluid (CSF). Since then some overviews have assessed up- or down-regulated miRNAs in brain tissues, blood and CSF in many thousands of $A D$ patients, but there is still no general consensus [6-8]. We must first be certain that miRNAs are really up- or down-regulated in $A D$ before using miRNAs as therapeutic methods. In human diseases there are three kinds of mature miRNA classes: up-regulated miRNAs, down-regulated miRNAs and "de novo" miRNAs whose new expression correlates with the "onset" or "emerging presence" of the disease state $[6,7]$. But no "de novo" miRNA has been reported in AD with the initiation or onset of the disease. Recently, some studies have demonstrated that in the early stages of AD there were dysregulated miRNAs and these molecules targeted multiple genes to alter key signaling pathways in the disease, which may ultimately modulate cell death mechanisms.

$A D$ is essentially human-specific, so the evaluation of human material must be included in research of miRNA-AD. Most research has evalu- ated human brain miRNAs involving gene expression profiling and assessed the levels of multiple miRNAs across different parts of brain samples [9-12]. But some tissue sampling protocols did not segregate white matter from gray matter, which increased variability. This caused different cell populations and little agreement between the differentially expressed gene lists in different protocols. Despite there being more and more evidence of miRNAs for AD pathology, dysregulation of miRNAs is not totally in accordance in peripheral blood levels and brain levels, especially in the same individuals, but studies often suffered from small sample size, heterogeneous designs, and conflicting outcomes. Because of the unclear pathogenesis of $A D$ and a lack of sensitive biomarkers, the $A D$ diagnosis still depends on progressive occurrence of the disease. In this study, we want to evaluate the dysregulation of microRNAs related to $A D$ to reveal the underlying complex mechanisms.

\section{Material and methods}

We aimed at defined objectives to collect all related miRNA, through extracting and analyzing data based on search strategy and inclusion/ exclusion criteria in our study protocol. We completed the present study in accordance with the Preferred Reporting Items for Systematic Reviews statement [13].

\section{Search strategy}

We used controlled vocabulary and text words to search using Ovid MEDLINE, Ovid EMBASE, PubMed, Google Scholar, Cochrane Central Register of Controlled Trials (CCTR), Cochrane Database of Systematic Reviews (CDSR), ACP Journal Club, Database of Abstracts of Review of Effectiveness (DARE) and Scopus and Web of Science, from January $1^{\text {st }} 2007$ to June $1^{\text {st }}, 2017$. Except MEDLINE, which uses the single term MicroRNAs, others use the terms MicroRNA, mir, mirna*, microrna* for individual miRNAs. The same approach for searching the databases of $A D$ was as follows: $A D$ is used by MEDLINE, but others use Alzheimer's disease, brain, cortex, cerebrospinal fluid and peripheral blood. We used EndNote (EndNote X7, Bld 7072, Thomson Research Soft, Stamford) to help us download all of the databases and remove duplicates. No language restrictions were applied for searching.

\section{Selection criteria}

No linguistic restrictions to avoid language bias resulted in outcomes limitations. We collected all related articles based on the inclusion and exclusion criteria. Two investigators respectively reviewed the collected titles and abstracts and 
separately continued to evaluate the full text of potentially eligible articles. Then two investigators put their results together to resolve disagreement about eligibility. In the next step, our co-authors further assessed the risk of trial bias and finally reached a consensus on each criterion (eligible case-control studies for the present study included those which investigated miRNAs' differential expression in brain, CSF or heparin-anticoagulated peripheral blood of AD patients and/or age- and gender-matched normal controls as inclusion criteria and case reports, conference presentations, editorials, and expert opinions as exclusion criteria). The miRNAs were identified through microarray analysis.

\section{Data extraction}

We extracted data from the included studies and listed them using the standardized datasheet. We respectively recorded expression profiles of miRNAs in brain, CSF or heparin-anticoagulated peripheral blood of AD patients and/or age- and gender-matched normal controls. In the datasheet, we listed the related miRNAs, upregulation vs. downregulation expression, the experimental models, and samples used. We also collected the experimentally verified miRNAs involving $A D$ and listed them including miRNAs, their targets, functions, and sample used.

\section{Applied bioinformatic analysis of miRNAs linked to $A D$}

Through Venny diagrams (Venny; http://bioinfogp.cnb.csic.es/tools/venny/), the Functional Enrichment analysis tool (FunRich; http://www. funrich.org/) and hierarchical clustering (GENE-E; https://software.broadinstitute.org/GENE-E/), we assessed common miRNAs dysregulated in the AD in different samples such as in brain, CSF or heparin-anticoagulated peripheral blood of AD patients and/or age- and gender-matched normal controls. Then we respectively analyzed common sets of AD-related miRNAs in brain, CSF or heparin-anticoagulated peripheral blood and investigated the relatedness of molecular findings on miRNAs in different studies using Heatmap analysis. We generated a matrix from multiple studies and common miRNAs for multiple comparisons, which was assigned a value of 1 if the miRNA was detected, while a value of 0 was assigned if miRNAs were not reported. Then through TargetScan (TargetScan Human Prediction of microRNA targets; http://www.targetscan.org/vert_71/) we accessed putative targets of different sets of miRNAs and through DAVID (The Database for Annotation, Visualization and Integrated Discovery; https://david.ncifcrf.gov/), the Functional Enrichment analysis tool (FunRich; http://www.funrich.org/), and String (http://string-db.org/) we investigated the relatedness of these gene targets in cellular networks by gene ontology analysis.

\section{Results}

\section{Study characteristics}

After repeated screening with inclusion and exclusion criteria we obtained the related studies of miRNA-AD association (Figure 1). The first screening obtained 3316 references that related to miRNA dysregulation. The second removed 2980 references based on titles and abstracts which did not mention actual concrete data (Figure 1). Because they lacked controls or assessment of miRNA dysregulation, 308 candidate studies were omitted (Figure 1). Finally, we obtained 28 suitable studies to perform statistics of the relationship between miRNAs and AD (Tables I and II).

Database search strategy

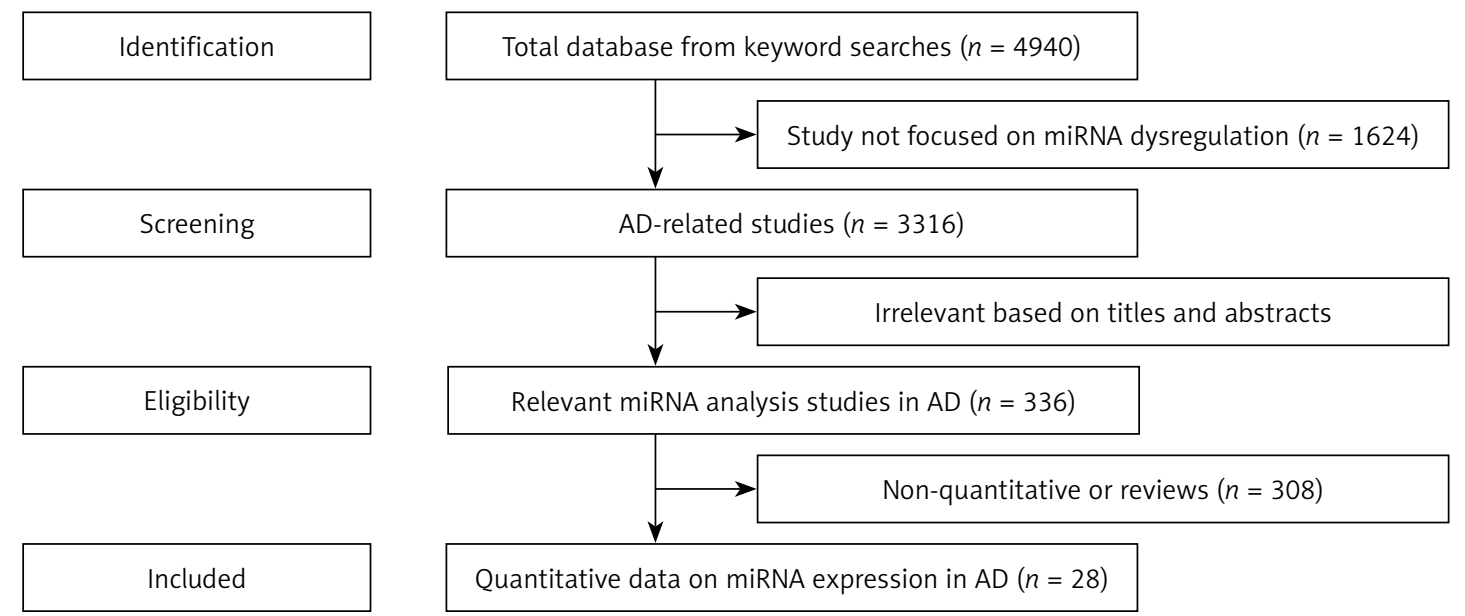

Figure 1. Flow diagram shows the current system of review, identification, screening, inclusion and exclusion 
Table I. Experimentally verified miRNAs involving AD

\begin{tabular}{|c|c|c|c|c|c|}
\hline miRNAs & Known targets & Tissues & & Functions & References \\
\hline \multirow[t]{2}{*}{ miR-7 } & \multirow[t]{2}{*}{ UBE2A, TSPAN-12 } & Cortex & $\downarrow$ & \multirow[t]{2}{*}{ Amyloidogenesis } & 6 \\
\hline & & Cortex & $\uparrow$ & & $1,16,24$ \\
\hline \multirow[t]{4}{*}{ miR-9 } & \multirow{4}{*}{$\begin{array}{l}\text { CFH, TREM2, SYNJ1, SYNPR, } \\
\text { GMEB2, TGFBI, FGFR1, NFKB, } \\
\text { SIRT1, TRIM2, BTBD3 }\end{array}$} & Blood & $\uparrow$ & \multirow{4}{*}{$\begin{array}{c}\text { Inflammation } \\
\text { Amyloid- } \beta \text { deposition }\end{array}$} & 24,25 \\
\hline & & Blood & $\downarrow$ & & 18 \\
\hline & & Cortex & $\downarrow$ & & $1,6,12,26$ \\
\hline & & Cortex & $\uparrow$ & & 24 \\
\hline miR-26b & $\mathrm{Rb} 1$ & Cortex & $\uparrow$ & $\begin{array}{l}\text { Activates cell cycle entry } \\
\text { Tau-phosphorylation }\end{array}$ & 11,27 \\
\hline \multirow[t]{2}{*}{$\operatorname{miR}-29(a / b)$} & \multirow[t]{2}{*}{ hBACE1 } & $\begin{array}{l}\text { Cortex } \\
\text { Cerebellum } \\
\text { Blood } \\
\end{array}$ & $\downarrow$ & \multirow[t]{2}{*}{$\begin{array}{l}\text { Neuronal maturation and } \\
\text { apoptosis }\end{array}$} & $28-31$ \\
\hline & & Blood & $\uparrow$ & & 23 \\
\hline miR-29c & BACE1 & Brain & $\downarrow$ & $\begin{array}{c}\text { Neuronal maturation and } \\
\text { apoptosis }\end{array}$ & 32 \\
\hline miR-34a & $\begin{array}{l}\text { TREM2, NDUFC2, SDHC, } \\
\text { UQCRB, UQCRQ, COX 10, } \\
\text { H6PD, PFK1, PFK2, LDH }\end{array}$ & Brain & $\uparrow$ & $\begin{array}{l}\text { A } \beta 42 \text { peptide clearance } \\
\text { activates cell cycle entry }\end{array}$ & $19,33,34$ \\
\hline $\mathrm{miR}-34 \mathrm{~b} / \mathrm{c}$ & $\mathrm{BCL2}$ & Brain & $\uparrow$ & $\begin{array}{l}\text { Neuronal maturation } \\
\text { Tau-phosphorylation }\end{array}$ & 35,36 \\
\hline miR-98-5p & SNX6 & Brain & $\downarrow$ & Amyloid- $\beta$ deposition & 37 \\
\hline miR-106 & ABCA1 & $\begin{array}{l}\text { Cortex } \\
\text { Blood }\end{array}$ & $\downarrow$ & Amyloid- $\beta$ deposition & 38,39 \\
\hline miR-107 & BACE1, CDK5, ADAM10 & Cortex & $\downarrow$ & Neuronal differentiation & $40-43$ \\
\hline miR-124 & BACE1 & Cortex & $\downarrow$ & Neuronal differentiation & 44 \\
\hline \multirow[t]{2}{*}{ miR-125b } & \multirow[t]{2}{*}{$\begin{array}{l}\text { 15-LOX, CFH, IKBKG, SYN-2, } \\
\text { TREM2, VDR, CDKN2A }\end{array}$} & $\begin{array}{l}\text { CSF } \\
\text { Brain }\end{array}$ & $\uparrow$ & \multirow{2}{*}{$\begin{array}{l}\text { Inflammation } \\
\text { Neurotrophism } \\
\text { Synaptogenesis }\end{array}$} & 24,45 \\
\hline & & Blood & $\downarrow$ & & 25,46 \\
\hline miR-128 & TFEB, GLB1, HEXA, CTSB & Blood & $\uparrow$ & $\begin{array}{l}\text { Neuronal differentiation } \\
\text { Amyloid- } \beta \text { deposition }\end{array}$ & 47 \\
\hline miR-132 & s ERK1, ERK2, GSK3b, and TAU & Brain & $\downarrow$ & $\begin{array}{l}\text { Inflammation } \\
\text { Memory-promoting } \\
\text { Amyloidogenesis }\end{array}$ & $48-5051$ \\
\hline miR-134 & - & CSF & $\uparrow$ & Synaptogenesis & 52 \\
\hline miR-137 & SPT & Cortex & $\downarrow$ & $\begin{array}{l}\text { Neuronal differentiation } \\
\text { Morphogenesis }\end{array}$ & 53 \\
\hline miR-144 & ADAM10 & Brain & $\uparrow$ & Amyloid- $\beta$ deposition & 54 \\
\hline miR-146a & CFH, IRAK- 1, TSPAN- 12 & $\begin{array}{l}\text { Cortex, } \\
\text { Hippocam- } \\
\text { pus }\end{array}$ & $\uparrow$ & $\begin{array}{l}\text { Amyloidogenesis } \\
\text { Inflammation }\end{array}$ & $16,24,55-58$ \\
\hline miR-153 & APP, APLP2 & Cortex & $\downarrow$ & Amyloidogenesis & 59 \\
\hline miR-155 & CFH & Cortex & $\uparrow$ & Inflammation & 16,24 \\
\hline miR-181 & SIRT1, BTBD3, TRIM2 & $\begin{array}{l}\text { Cortex } \\
\text { Blood }\end{array}$ & $\downarrow$ & Amyloid- $\beta$ deposition & 25,26 \\
\hline miR-193b & APP & $\begin{array}{l}\text { Blood } \\
\text { CSF }\end{array}$ & $\downarrow$ & Neurons degradation & 60 \\
\hline miR-210 & VEGF & $\begin{array}{l}\text { Blood } \\
\text { CSF }\end{array}$ & $\downarrow$ & Neuronal differentiation & 61 \\
\hline miR-211-5p & NUAK1 & Cortex & $\uparrow$ & $\begin{array}{l}\text { Neuronal differentiation } \\
\text { Amyloid- } \beta \text { deposition }\end{array}$ & 62 \\
\hline miR-339-5p & BACE1 & Brain & $\downarrow$ & Amyloid- $\beta$ deposition & 63 \\
\hline miR-384 & APP, BACE-1 & $\begin{array}{l}\text { Blood } \\
\text { CSF }\end{array}$ & $\downarrow$ & $\begin{array}{l}\text { Neurons degradation } \\
\text { activates cell cycle entry } \\
\text { synaptogenesis }\end{array}$ & 64 \\
\hline miR-512 & cFLIP and MCL1 & Brain & $\downarrow$ & Tau-phosphorylation & 20 \\
\hline
\end{tabular}

ADAM 10 - adisinteg- rinandmetalloprotease 10, APLP2 - amyloid precursor-like protein 2, BACE1 - $\beta$-site amyloid precursor proteincleaving enzyme 1, BDNF - brain-derived neurotrophic factor, BTBD3 - BTB domain containing 3, CDKN2A - cell cycle regulator, GMEB2 - glucocorticoid-sensing factor, hBACE1 - high levels of human $\beta$-secretase, Rb 1-retinoblastoma protein, SIRT1 - sirtuin 1, SNX6 - sorting nexin 6, SYN-2 - synaptic phosphoprotein, SYNJ1 - synaptojanin 1, SYNPR - synaptoporin, TGFBI - transforming growth factor, TRIM2 tripartitemotif-containing 2, VDR - the vitamin D3 receptor, VEGF - vascular endothelial growth factor, 15-LOX - 15-lipoxygenase. 
Table II. Expression profiles of miRNAs in AD

\begin{tabular}{|c|c|c|}
\hline Reference & miRNAs & $\begin{array}{l}\text { Experimental } \\
\text { models and } \\
\text { sample size }\end{array}$ \\
\hline $\begin{array}{l}\text { Pichler } \\
2017^{6} \\
\text { Cortex Up }\end{array}$ & $\begin{array}{l}\text { miR-1274b, miR-664, miR-143, miR-195, miR-876-5p, miR-4270, miR-296-3p, } \\
\text { miR-1972, miR-1324, miR-152, miR-3202, miR-1246, miR-500a, miR-92a-2*, } \\
\text { miR-762, miR-3621, miR-519d, miR-517b, miR-3605-5p, miR-3141, miR-3941, } \\
\text { miR-183*, miR-196b*, miR-30c, miR-3909, miR-943, miR-548t, miR-3622b-5p, } \\
\text { miR-550a, miR-1228*, miR-2117, miR-423-5p, miR-302c, miR-675, miR-301b, } \\
\text { miR-514b-3p, miR-1293, miR-608, miR-149*, miR-3911, miR-3133 }\end{array}$ & \multirow[t]{2}{*}{$\begin{array}{l}39 \text { AD patients } \\
\text { and } 25 \text { age- } \\
\text { matched } \\
\text { healthy control }\end{array}$} \\
\hline $\begin{array}{l}\text { Pichler } \\
2017^{6} \\
\text { Cortex Down }\end{array}$ & 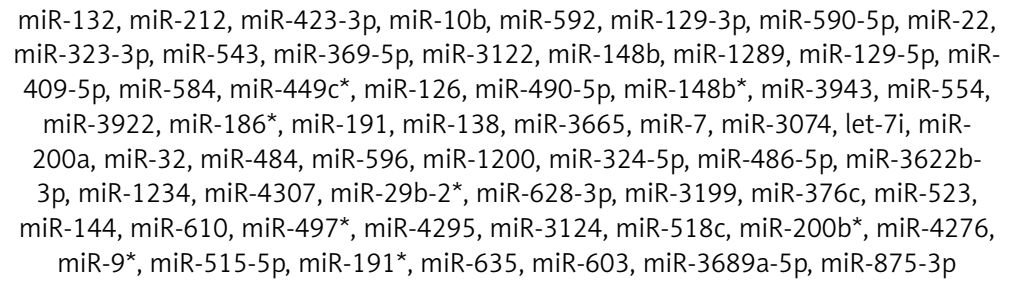 & \\
\hline $\begin{array}{l}\text { Nagaraj } 2017^{7} \\
\text { Blood Up }\end{array}$ & $\begin{array}{l}\text { miR-486-5p, miR-33a-5p(MCI-AD2), miR-483-5p, miR-320a, miR-320b, miR-320c, } \\
\text { miR-502-3p, miR-200a-3p, miR-1260a }\end{array}$ & \multirow{2}{*}{$\begin{array}{l}35 \text { AD patients } \\
\text { and } 15 \text { age- } \\
\text { matched } \\
\text { healthy control }\end{array}$} \\
\hline $\begin{array}{l}\text { Nagaraj } 2017^{7} \\
\text { Blood Down }\end{array}$ & $\begin{array}{l}\text { miR-151a-5p, miR-30b-5p, miR-33a-5p, miR-18a-5p, miR-320c, miR-103a-3p, miR- } \\
\text { 301a-3p, miR-142-3p }\end{array}$ & \\
\hline $\begin{array}{l}\text { Hara } 2017^{11} \\
\text { Blood Up }\end{array}$ & miR-26b-5p, let-7f-5p, miR-32-5p, let-7a-5p, let-7c-5p, miR-140-3p & \multirow{3}{*}{$\begin{array}{l}27 \text { AD patients } \\
\text { and } 18 \text { age- } \\
\text { matched } \\
\text { healthy control }\end{array}$} \\
\hline $\begin{array}{l}\text { Hara } 2017^{11} \\
\text { Blood Down }\end{array}$ & $\begin{array}{c}\text { miR-501-3p, miR-151a-3p, miR-146a-5p, miR-148a-3p, miR-100-5p, miR-183-5p, } \\
\text { miR-186-5p, miR-342-3p, miR-4485, miR-486-3p, miR-500a-3p, miR-502-3p, miR- } \\
\text { 874-3p, miR-98-5p }\end{array}$ & \\
\hline $\begin{array}{l}\text { Hara } 2017^{11} \\
\text { Cortex Up }\end{array}$ & $\begin{array}{l}\text { hsa-miR-1307-3p, hsa-miR-342-3p, hsa-miR-193b-5p, hsa-miR-671-3p, hsa-miR- } \\
\text { 330-3p, hsa-miR-193a-5p, hsa-miR-187-3p, hsa-miR-873-3p, hsa-miR-139-5p, } \\
\text { hsa-miR-550a-3p, hsa-miR-346, hsa-miR-1224-5p, hsa-miR-769-3p, hsa-miR-324- } \\
\text { 3p, hsa-miR-1226-3p, hsa-miR-2110, hsa-miR-1237-3p, hsa-miR-125b-5p, hsa- } \\
\text { miR-3622a-3p, hsa-miR-1229-3p, hsa-miR-328-3p, hsa-miR-1976, hsa-miR-484, } \\
\text { hsa-miR-487a-5p, hsa-miR-125a-5p, hsa-miR-1227-3p, hsa-miR-25-5p, hsa-miR- } \\
\text { 6894-3p, hsa-miR-760, hsa-miR-6760-3p, hsa-miR-197-3p, hsa-miR-1910-5p, hsa- } \\
\text { miR-532-3p, hsa-miR-423-3p, hsa-miR-935, hsa-miR-6775-3p, hsa-miR-504-5p, } \\
\text { hsa-miR-6737-3p, hsa-miR-887-5p, hsa-miR-6798-3p, hsa-miR-6756-3p, hsa-miR- } \\
\text { 4646-3p, hsa-miR-1301-3p, hsa-miR-3180-5p, hsa-miR-940, hsa-miR-7114-3p, } \\
\text { hsa-miR-1306-5p, hsa-miR-326, hsa-miR-3177-3p, hsa-miR-6794-3p, hsa-miR- } \\
\text { 6747-3p, hsa-miR-149-5p, hsa-miR-1228-3p, hsa-miR-100-5p, hsa-miR-485-5p, } \\
\text { hsa-miR-3615, hsa-miR-92b-3p, hsa-miR-331-3p, hsa-miR-370-3p, hsa-miR- } \\
\text { 4787-3p, hsa-miR-210-5p, hsa-miR-128-2-5p, hsa-miR-296-5p, hsa-miR-1260b, } \\
\text { hsa-miR-744-5p, hsa-miR-3940-3p, hsa-miR-1260a, hsa-miR-1224-3p, hsa-miR- } \\
\text { 6803-3p, hsa-miR-6511a-3p, hsa-miR-323a-5p, hsa-miR-6511b-3p, hsa-miR-766- } \\
\text { 3p, hsa-miR-6726-3p, hsa-miR-1908-5p, hsa-miR-1247-5p, hsa-miR-1180-3p, hsa- } \\
\text { miR-7704, hsa-miR-92b-5p, hsa-miR-1343-3p, hsa-miR-204-3p, hsa-miR-877-5p, } \\
\text { hsa-miR-4510, hsa-miR-885-3p, hsa-miR-1249, hsa-miR-504-3p, hsa-miR-99b-5p, } \\
\text { hsa-miR-1271-5p, hsa-miR-501-3p, hsa-miR-5100, hsa-miR-409-5p }\end{array}$ & \\
\hline $\begin{array}{l}\text { Hara } 2017^{11} \\
\text { Cortex Down }\end{array}$ & $\begin{array}{l}\text { miR-146b-5p, miR-6087, miR-223-3p, miR-378h, miR-21-5p, miR-146a-5p, miR- } \\
\text { 10b-5p, miR-132-3p, miR-23a-3p, miR-660-5p, miR-23b-3p, miR-143-3p, miR-582- } \\
\text { 3p, miR-576-5p, miR-126-3p, miR-135a-5p, miR-132-5p, miR-151a-3p, miR-28-3p, } \\
\text { miR-454-3p, miR-186-5p, miR-374b-5p, miR-889-3p, miR-130a-3p, miR-582-5p, } \\
\text { miR-629-5p, miR-155-5p, miR-3609, miR-142-5p, miR-182-5p, miR-218-5p, } \\
\text { miR-9-3p, miR-203a, miR-9-5p, let-7a-3p, miR-26a-5p, miR-192-5p, miR-379-3p, } \\
\text { miR-181a-3p, miR-598-3p, miR-19b-3p, miR-7-5p, miR-4461, miR-496, miR-412- } \\
\text { 5p, miR-136-3p, miR-130b-3p, miR-184, miR-30e-5p, miR-194-5p, miR-7-1-3p, } \\
\text { miR-532-5p, miR-183-5p, miR-335-3p, miR-215-5p, miR-7-2-3p, miR-212-3p, miR- } \\
\text { 27b-5p, miR-378a-3p, miR-26b-5p, miR-301a-3p, let-7i-5p, miR-16-5p, miR-152- } \\
\text { 3p, miR-541-3p, miR-148a-3p, miR-30e-3p, miR-148b-3p, miR-27b-3p, miR-421, } \\
\text { miR-27a-3p, miR-107, miR-24-3p, miR-103a-3p, miR-22-3p, miR-379-5p, miR-411- } \\
\text { 5p, miR-299-5p, miR-30a-5p, miR-98-5p, miR-25-3p, miR-30b-5p, miR-140-3p, } \\
\text { miR-495-3p, miR-2682-5p, miR-93-5p, miR-128-3p, let-7f-5p, miR-191-5p, miR- } \\
\text { 378g, miR-124-5p, miR-99a-3p, miR-30a-3p, miR-129-5p, miR-378c, miR-425-5p, } \\
\text { miR-181d-5p, miR-381-3p, miR-378d, miR-3117-3p, miR-181c-3p, miR-363-3p, } \\
\text { miR-30c-2-3p, miR-370-5p, miR-501-5p, miR-146b-3p, miR-6837-3p, miR-769-5p, } \\
\text { miR-30c-5p, miR-365a-3p, miR-365b-3p, miR-362-5p, miR-30d-5p, miR-3127-5p, } \\
\text { miR-3605-5p, miR-134-5p, miR-432-5p, miR-3200-3p, miR-127-5p, miR-409-3p, } \\
\text { miR-361-5p, miR-127-3p }\end{array}$ & $\begin{array}{l}27 \text { AD patients } \\
\text { and } 18 \text { age- } \\
\text { matched } \\
\text { healthy control }\end{array}$ \\
\hline
\end{tabular}


Table II. Cont.

\begin{tabular}{|c|c|c|}
\hline Reference & miRNAs & $\begin{array}{l}\text { Experimental } \\
\text { models and } \\
\text { sample size }\end{array}$ \\
\hline $\begin{array}{l}\text { Villela } 20166^{12} \\
\text { Cortex Down }\end{array}$ & miR-146b, miR-451, miR-9, miR-181c & $\begin{array}{l}10 \text { AD patients } \\
\text { and } 10 \text { age- } \\
\text { matched } \\
\text { healthy control }\end{array}$ \\
\hline $\begin{array}{l}\text { Sorensen } \\
2016^{13} \\
\text { CSF Up }\end{array}$ & let-7i-5p, miR-15a-5p & \multirow{4}{*}{$\begin{array}{l}10 \text { AD patients } \\
\text { and } 10 \text { age- } \\
\text { matched } \\
\text { healthy control }\end{array}$} \\
\hline $\begin{array}{l}\text { Sorensen } \\
2016^{13} \\
\text { CSF Down }\end{array}$ & $\operatorname{miR}-29 c-3 p$ & \\
\hline $\begin{array}{l}\text { Sorensen } \\
2016^{13} \\
\text { Blood Up }\end{array}$ & miR-590-5p, miR-142-5p & \\
\hline $\begin{array}{l}\text { Sorensen } \\
2016^{13} \\
\text { Blood Down }\end{array}$ & miR-194-5p & \\
\hline $\begin{array}{l}\text { Dangla } \\
2016^{14} \\
\text { CSF Up }\end{array}$ & miR-222, miR-125b & $\begin{array}{l}69 \text { AD patients } \\
\text { and } 69 \text { age- } \\
\text { matched } \\
\text { healthy control }\end{array}$ \\
\hline $\begin{array}{l}\text { Satoh } 2015^{5} \\
\text { Blood Up }\end{array}$ & $\begin{array}{l}\text { miR-26b-3p, miR-28-3p, miR-30c-5p, miR-30d-5p, miR-148b-5p, , miR-151a-3p, } \\
\text { miR-186-5p, miR-425-5p, , miR-550a-5p, miR-1468, miR-4781-3p, miR-5001-3p, } \\
\text { and miR-6513-3p }\end{array}$ & \multirow{2}{*}{$\begin{array}{l}48 \text { AD patients } \\
\text { and } 22 \text { age- } \\
\text { matched } \\
\text { healthy control }\end{array}$} \\
\hline $\begin{array}{l}\text { Satoh } 2015^{5} \\
\text { Blood Down }\end{array}$ & $\begin{array}{l}\text { let-7a-5p, let-7e-5p, let-7f-5p, let-7 g-5p, miR-15a-5p, miR-17-3p, , miR-29b-3p, } \\
\text { miR-98-5p, miR-144-5p, , miR-148a-3p, miR-502-3p, miR-660-5p, , miR-1294, and } \\
\text { miR-3200-3p }\end{array}$ & \\
\hline $\begin{array}{l}\text { Zhao } 2015^{24} \\
\text { Cortex Up }\end{array}$ & miR-7, miR-9, miR-34a, miR-125b, miR-146a, miR-155 & N/A \\
\hline $\begin{array}{l}\text { Mezache } \\
2015^{20} \\
\text { Cortex Down }\end{array}$ & miR-512, miR-765, miR-1181, miR-1292 & $\begin{array}{l}10 \text { AD patients } \\
\text { and } 10 \text { age- } \\
\text { matched } \\
\text { healthy control }\end{array}$ \\
\hline $\begin{array}{l}\text { Lei } 2015^{32} \\
\text { Cortex Down }\end{array}$ & $\operatorname{miR}-29 c$ & $\begin{array}{l}31 \text { AD patients } \\
\text { and } 29 \text { age- } \\
\text { matched } \\
\text { healthy control }\end{array}$ \\
\hline $\begin{array}{l}\text { Dong } 2015{ }^{65} \\
\text { Blood Down }\end{array}$ & miR-31, miR-93, miR-143, miR-146a, miR-148a, and miR-191 & $\begin{array}{l}127 \text { AD patients } \\
\text { and } 123 \\
\text { age-matched } \\
\text { healthy control }\end{array}$ \\
\hline $\begin{array}{l}\text { Denk } 2015^{66} \\
\text { CSF Up }\end{array}$ & miR-100, miR-146a, miR-296, miR-3622b-3p, miR-4467, miR-505*, miR-766 & \multirow{2}{*}{$\begin{array}{l}22 \text { AD patients } \\
\text { and } 28 \text { age- } \\
\text { matched } \\
\text { healthy control }\end{array}$} \\
\hline $\begin{array}{l}\text { Denk } 2015^{66} \\
\text { CSF Down }\end{array}$ & miR-103, miR-219, miR-335, miR-375, miR-708, miR-1274A, miR-4449, miR-4674 & \\
\hline $\begin{array}{l}\text { Tan } 2014^{15} \\
\text { Blood Up }\end{array}$ & miR-9 & \multirow{2}{*}{$\begin{array}{c}105 \text { AD patients } \\
\text { and } 150 \\
\text { age-matched } \\
\text { healthy control }\end{array}$} \\
\hline $\begin{array}{l}\text { Tan } 2014{ }^{15} \\
\text { Blood Down }\end{array}$ & miR-125b, miR-181c & \\
\hline $\begin{array}{l}\text { Kiko } 2014{ }^{23} \\
\text { Blood Down }\end{array}$ & miR-34a and miR-146a & \multirow[t]{3}{*}{ N/A } \\
\hline $\begin{array}{l}\text { Kiko } 2014^{23} \\
\text { Blood and } \\
\text { CSF Up }\end{array}$ & miR-29a, miR-29b & \\
\hline $\begin{array}{l}\text { Kiko } 2014^{23} \\
\text { CSF Down }\end{array}$ & miR-34a, miR-125b, and miR-146a & \\
\hline
\end{tabular}


Table II. Cont.

\begin{tabular}{|c|c|c|}
\hline Reference & miRNAs & $\begin{array}{l}\text { Experimental } \\
\text { models and } \\
\text { sample size }\end{array}$ \\
\hline $\begin{array}{l}\text { Galimberti } \\
2014^{67} \\
\text { Blood Down }\end{array}$ & miR-125b, miR-23a, miR-26b & \multirow{2}{*}{$\begin{array}{l}22 \text { AD patients } \\
\text { and } 10 \text { age- } \\
\text { matched } \\
\text { healthy control }\end{array}$} \\
\hline $\begin{array}{l}\text { Galimberti } \\
2014^{67} \\
\text { CSF Down }\end{array}$ & miR-125b, miR-26b & \\
\hline $\begin{array}{l}\text { Lau } 2013^{8} \\
\text { Cortex Up }\end{array}$ & $\begin{array}{l}\text { miR-92b-3p, miR-874, miR-744-5p, miR-519a-3p, miR-517c-3p, miR-455-5p, miR- } \\
\text { 424-5p, miR-363-3p, miR-362-3p, miR-27a-3p, miR-23a-3p, miR-223-3p, miR-214- } \\
\text { 3p, miR-200a-3p, miR-199b-3p, miR-199a-3p, miR-195-5p, miR-190b, miR-150-5p, } \\
\text { miR-142-3p, miR-1275, miR-1260a, let-7i-5p, let-7f-5p }\end{array}$ & \multirow[t]{2}{*}{$\begin{array}{l}41 \text { AD patients } \\
\text { and } 23 \text { age- } \\
\text { matched } \\
\text { healthy control }\end{array}$} \\
\hline $\begin{array}{l}\text { Lau } 2013^{8} \\
\text { Cortex Down }\end{array}$ & $\begin{array}{l}\text { miR-758-3p, miR-633, miR-551b-3p, miR-548j, miR-520a-3p, miR-508-3p, miR- } \\
\text { 496, miR-491-3p, miR-487a, miR-485-5p, miR-431-5p, miR-421, miR-409-5p, miR- } \\
\text { 382-5p, miR-370, miR-337-3p, miR-219-1-3p, miR-210, miR-138-5p, miR-136-5p, } \\
\text { miR-135b-5p, miR-133b, miR-132-3p, miR-1321, miR-129-5p, miR-129-2-3p, } \\
\text { miR-128, miR-127-5p, miR-124-3p, miR-1179, miR-1178-3p, miR-10b-5p }\end{array}$ & \\
\hline $\begin{array}{l}\text { Smith } \\
2013^{68} \\
\text { Cortex Up }\end{array}$ & $\begin{array}{l}\operatorname{miR}-112, \operatorname{miR}-161 \text {, let-7d-3p, miR-5010-3p, miR-26a-5p, miR-1285-5p, miR-151a- } \\
3 p\end{array}$ & \multirow[t]{2}{*}{ N/A } \\
\hline $\begin{array}{l}\text { Smith } \\
2013^{68} \\
\text { Cortex Down }\end{array}$ & miR-103a-3p, miR-107, miR-532-5p, miR-26b-5p, let-7f-5p & \\
\hline $\begin{array}{l}\text { Leidinger } \\
2013^{21} \\
\text { Blood Up }\end{array}$ & $\begin{array}{c}\text { miR-99b-5p, miR-125a-5p, let-7b-3p, let-7f-1-3p, let-7d-3p, miR-30c-5p, miR- } \\
\text { 30a-3p, miR-30a-5p, miR-112, miR-161, miR-5010-3p, miR-26a-5p, miR-1285-5p, } \\
\text { miR-151a-3p }\end{array}$ & \multirow{2}{*}{$\begin{array}{l}215 \text { AD patients } \\
\text { and } 215 \\
\text { age-matched } \\
\text { healthy control }\end{array}$} \\
\hline $\begin{array}{l}\text { Leidinger } \\
2013^{21} \\
\text { Blood Down }\end{array}$ & $\begin{array}{l}\text { let-7e-5p, let-7a-5p, let-7b-5p, miR-103a-3p, miR-107, miR-532-5p, miR-26b-5p, } \\
\text { let-7f-5p }\end{array}$ & \\
\hline $\begin{array}{l}\text { Kumar } \\
2013^{69} \\
\text { Blood Up }\end{array}$ & miR-301a-3p, miR-545-3p & \multirow{2}{*}{$\begin{array}{c}20 \text { AD patients } \\
\text { and } 20 \text { age- } \\
\text { matched } \\
\text { healthy control }\end{array}$} \\
\hline $\begin{array}{l}\text { Kumar } \\
2013^{69} \\
\text { Blood Down }\end{array}$ & miR-191-5p, miR-15b-5p, let-7d-5p, let-7g-5p, miR-142-3p & \\
\hline $\begin{array}{l}\text { Geekiyanage } \\
2012^{31} \\
\text { Blood Down }\end{array}$ & miR-137, miR-181c, miR-9, miR-29a and miR-29b & $\begin{array}{l}7 \text { AD patients } \\
\text { and } 7 \text { age- } \\
\text { matched } \\
\text { healthy control }\end{array}$ \\
\hline $\begin{array}{l}\text { Alexandrov } \\
2012^{70} \\
\text { CSF Up }\end{array}$ & miRNA-9, miRNA-125b, miRNA-146a, miRNA-155 & N/A \\
\hline $\begin{array}{l}\text { Wang } 2011^{9} \\
\text { White matter } \\
\text { Up }\end{array}$ & miR-215, miR-509-5p, miR-574-3p, miR-576-5p, miR-302e, miR-220b, miR-208a & \multirow{2}{*}{$\begin{array}{c}6 \text { AD patients } \\
\text { and } 4 \text { age- } \\
\text { matched } \\
\text { healthy control }\end{array}$} \\
\hline $\begin{array}{l}\text { Wang } 2011^{9} \\
\text { White matter } \\
\text { Down }\end{array}$ & $\begin{array}{l}\text { miR-491-3p, miR-17, miR-339-5p, miR-320d, let-7a, miR-26a, let-7f, miR-27a, } \\
\text { miR-190, miR-423-5p, miR-219-5p, miR-181b, miR-106a, miR-194, miR-193b, } \\
\text { miR-320b, miR-374b, miR-513a-5p, miR-520d-5p, miR-223, miR-589, miR-301a, } \\
\text { miR-34b, miR-338-3p, let-7b, miR-32, let-7c, miR-106b, miR-320c, miR-140- } \\
\text { 3p, miR-212, miR-193a-3p, miR-412, let-7i, miR-365, miR-422a, miR-219-2-3p, } \\
\text { miR-151-3p, miR-340, miR-330-3p, miR-16, miR-320a, miR-374a, miR-142-5p, } \\
\text { miR-92b, miR-185, miR-361-3p, miR-23a, miR-34c-5p, miR-338-5p, miR-197, miR- } \\
\text { 19b, miR-27b, miR-23b, miR-557, miR-24, miR-142-3p, miR-330-5p, miR-148b, } \\
\text { miR-361-5p, miR-363, miR-584, miR-181a, miR-19a, miR-21, miR-93, miR-15b, } \\
\text { miR-33a, miR-140-5p, miR-26b, miR-186, miR-101, miR-423-3p, miR-326, miR- } \\
\text { 20a, miR-151-5p, miR-15a, miR-99b }\end{array}$ & \\
\hline
\end{tabular}


Table II. Cont.

\begin{tabular}{|c|c|c|}
\hline Reference & miRNAs & $\begin{array}{l}\text { Experimental } \\
\text { models and } \\
\text { sample size }\end{array}$ \\
\hline $\begin{array}{l}\text { Wang } 2011^{9} \\
\text { Cortex Up }\end{array}$ & $\begin{array}{l}\text { miR-519e, miR-490-3p, miR-298, miR-214, miR-574-5p, miR-498, miR-518a-5p, miR- } \\
\text { 527, miR-525-5p, miR-300, miR-576-3p, miR-583, miR-146b-3p, miR-549, miR-516a- } \\
\text { 5p, miR-510, miR-184, miR-516b, miR-198, miR-451, miR-144, miR-424, let-7e }\end{array}$ & \multirow{2}{*}{$\begin{array}{l}6 \text { AD patients } \\
\text { and } 4 \text { age- } \\
\text { matched } \\
\text { healthy control }\end{array}$} \\
\hline $\begin{array}{l}\text { Wang } 2011^{9} \\
\text { Cortex Down }\end{array}$ & $\begin{array}{l}\text { miR-99a, miR-98, miR-95, miR-9, miR-7, miR-582-5p, miR-551b, miR-519d, miR- } \\
\text { 495, miR-491-5p, miR-488, miR-487b, miR-487a, miR-485-3p, miR-432, miR-425, } \\
\text { miR-411, miR-382, miR-381, miR-379, miR-378, miR-377, miR-376c, miR-34a, } \\
\text { miR-342-3p, miR-335, miR-331-3p, miR-329, miR-30e, miR-30d, miR-30c, miR-30b, } \\
\text { miR-30a, miR-29c, miR-29b, miR-29a, miR-299-5p, miR-222, miR-221, miR-22, } \\
\text { miR-218, miR-195, miR-191, miR-149, miR-146b-5p, miR-145, miR-143, miR-139- } \\
\text { 5p, miR-138, miR-136, miR-135a, miR-129-5p, miR-129-3p, miR-128, miR-127-5p, } \\
\text { miR-127-3p, miR-126, miR-125b, miR-125a-5p, miR-124, miR-107, miR-103, let-7g }\end{array}$ & \\
\hline $\begin{array}{l}\text { Nunez } \\
2010^{22} \\
\text { Cortex Up }\end{array}$ & $\begin{array}{l}\text { miR-601, mir-23974, miR-575, miR-765, mir-06383, miR-188, miR-671, mir-30184, } \\
\text { miR-617, mir-10912, mir-10939, mir-45605, miR-134, miR-320, mir-35456, mir- } \\
\text { 18895, miR-572, mir-28648, mir-19790, miR-486, miR-432, miR-382, miR-185 }\end{array}$ & \multirow{2}{*}{$\begin{array}{l}5 \text { AD patients } \\
\text { and } 5 \text { age- } \\
\text { matched } \\
\text { healthy control }\end{array}$} \\
\hline $\begin{array}{l}\text { Nunez } \\
2010^{22} \\
\text { Cortex Down }\end{array}$ & $\begin{array}{l}\text { miR-494, miR-598, miR-29c, miR-130a, miR-15a, miR-29b, mir-08570, miR-20b, } \\
\text { miR-101, 5S-b2, mir-42448, mir-02532, miR-148b, mir-20546, miR-95, miR-368, } \\
\text { miR-181c, mir-44608, miR-376a, miR-30e-5p, mir-12497, mir-12504, mir-05109, } \\
\text { miR-582, miR-374 }\end{array}$ & \\
\hline $\begin{array}{l}\text { Sethi } 2009^{3} \\
\text { Cortex Up }\end{array}$ & miRNA-9, miRNA-125b, miRNA-146a & $\mathrm{N} / \mathrm{A}$ \\
\hline $\begin{array}{l}\text { Hebert } \\
2008^{71} \\
\text { Cortex Up }\end{array}$ & miR-197, miR-511, miR-320 & \multirow[t]{2}{*}{ N/A } \\
\hline $\begin{array}{l}\text { Hebert } \\
2008^{71} \\
\text { Cortex Down }\end{array}$ & $\begin{array}{l}\text { miR-29a, miR-29b-1, miR-9, miR-210, miR-181c, miR-15a, miR-22, miR-101, miR- } \\
\text { 19b, let-7i, miR-106b, miR-26b, miR-363, miR-93 }\end{array}$ & \\
\hline $\begin{array}{l}\text { Cogswell } \\
2008^{1} \\
\text { Cortex Up }\end{array}$ & $\begin{array}{l}\text { miR-423, miR-29a, miR-29b, miR-145, miR-143, miR-148b, miR-7, miR-324-5p, } \\
\text { miR-519d }\end{array}$ & \multirow{3}{*}{$\begin{array}{l}15 \text { AD patients } \\
\text { and } 12 \text { age- } \\
\text { matched } \\
\text { healthy control }\end{array}$} \\
\hline $\begin{array}{l}\text { Cogswell } \\
2008^{1} \\
\text { Cortex Down }\end{array}$ & miR-9, miR-132, miR-212, miR-191, miR-146b & \\
\hline $\begin{array}{l}\text { Cogswell } \\
2008^{1} \\
\text { CSF Up and } \\
\text { Down }\end{array}$ & $\begin{array}{l}\text { let-7f, miR-105, miR-10a, miR-10b, miR-125a, miR-126, miR-126*, miR-127, miR- } \\
\text { 135a, miR-138, miR-141, miR-142-5p, miR-143, miR-146b, miR-151, miR-154, } \\
\text { miR-15b, miR-181a, miR-181c, miR-186, miR-191, miR-194, miR-195, miR-197, } \\
\text { miR-199a*, miR-204, miR-205, miR-214, miR-216, miR-221, miR-302b, miR-30a- } \\
\text { 3p, miR-30a-5p, miR-30b, miR-30c, miR-30d, miR-32, miR-338, miR-345, miR-362, } \\
\text { miR-371, miR-374, miR-375, miR-380-3p, miR-422b, miR-429, miR-448, miR-449, } \\
\text { miR-451, miR-455, miR-494, miR-497, miR-501, miR-517a, miR-517b, miR-518b, } \\
\text { miR-518f, miR-520a*, miR-526a, miR-99a }\end{array}$ & \\
\hline $\begin{array}{l}\text { Wang } \\
2008^{43} \\
\text { Cortex Down }\end{array}$ & miR-107, miR-23b & $\begin{array}{l}6 \text { AD patients } \\
\text { and } 11 \text { age- } \\
\text { matched } \\
\text { healthy control }\end{array}$ \\
\hline $\begin{array}{l}\text { Lukiw } 2007^{2} \\
\text { Cortex Up }\end{array}$ & miR-9, miR-124a, miR-125b, miR-128, miR-132, miR-219 & N/A \\
\hline
\end{tabular}

We used data search tools to assess the number of dysregulated miRNAs which have been experimentally verified to relate to AD pathology (Table I). There will be more and more evidence about miRNA-AD association over time. From our database 27 dysregulated miRNAs were identified as involved in amyloidogenesis, inflammation, degradation of neurons, neurite differentiation, antiapoptotic, neuronal survival and proliferation,
A $\beta$-induced pathologies, aberrant network activity and synaptic depression.

In our literature many miRNAs are dysregulated in $A D$ and even the same miRNAs in different studies are identified as differently up-regulated or down-regulated (Tables I and II). We used microarray analysis of AD specimens from the candidate studies to assess the implicated miRNAs with comparison to age-matched healthy control spec- 
imens. Originally, the studies primarily focused on dysregulation of miRNAs in cortex of AD [5] aimed at finding the pathology of AD. Recently there are still many studies which try to identify primary dysregulation of miRNAs in $A D[9,14,15]$. With the development of AD pathology, more and more studies have focused on dysregulation of miRNAs in blood or CSF of AD aimed at identifying the preclinical phase early $[10,14,16-18]$. In order to provide the first insights into the miRNAs' different expression between human cerebral cortical gray matter (GM) and white matter (WM) in $A D$, Wang et al. [12] identified a significant number of dysregulated miRNAs in GM and WM of AD brain but those data suggested that patterns of miRNA expression in cortical GM may contribute to $A D$ pathogenetically. Pichler et al. [9] found that miR-7 expression was downregulated in cortex, which correlated with $\mathrm{AD}$ amyloidogenesis. But Lukiw et al. [19] and Cogswell et al. [4] separately reported that miR-7 was upregulated in cortex. MiR-9 was also identified as differently dysregulated in $A D$, which was assessed as downregulated in cortex $[9,15,20]$ and in blood [21] but the Zhao et al. [22] study identified upregulation in cortex.

In our bioinformatics analysis, whether in cortex or in blood and CSF, a number of dysregulated miRNAs have been identified as up- or down-regulated in different studies [4, 8-11, 14, 17, 22-26]. These data separately showed overlapping miRNA expression for the first time, which resulted in significant problems (Table II). Even several miRNAs in CSF are reported as upregulated and downregulated targets in AD in the same class [4]. However, none of the studies reported the same upand down-regulated miRNAs from the same class (Figure 2).

We used clustering to generate a heatmap to visualize the different studies' relationship for the related set of miRNAs. More specifically, we compared the findings of 15 studies related to cortex, 11 studies related to blood and 7 studies related to CSF by aiming to identify miRNAs in AD. It is still mostly unknown what the biological and clinical distinctions and function are in the up-regulated and down-regulated miRNAs in different tissues. However, dysregulated miRNAs may play a crucial role in the development of $A D$ pathology as biomarkers and it is worth making a re-evaluation of the medical records of the miRNA in these. We analyzed either up- or down-regulated miRNAs coordinately modulated with the disease state and found that they have common functions and partly regulate common pathways. Therefore, we used TargetScan to search the predicted targets of each set of miRNAs and tabulated the number of mRNAs that match to a specific miRNA to perform clustering analysis in order to define how many common mRNA targets there are in different sets of miRNAs.

Preliminary Venny analysis by TargetScan and FunRich tools showed common targets of "Up-regulated miRNAs (miR-143, miR-519d, miR-7, miR92b-3p, miR-744-5p, miR-1260a)" and "Down-regulated miRNAs (let-7f-5p, miR-103a-3p, miR-107, miR-10b-5p, miR-127-5p, miR-129-5p, miR-132, miR-146b, miR-148b, miR-181c, miR-191, miR212, miR-26b-5p, miR-29c, miR-30e-5p, miR-4095p, miR-421, miR-496, miR-532-5p, miR-9)" in cortex (Figure 3), common targets of "dysregulated miRNAs (let-7a-5p, let-7e-5p, let-7f-5p, miR103a-3p, miR-142-3p, miR-146a, miR-148a-3p, miR-151a-3p, miR-181c, miR-186-5p, miR-26b-5p, miR-29a, miR-29b, miR-301a-3p, miR-30c-5p, miR502-3p, miR-9, miR-98-5p)" in blood (see above), and common targets of "dysregulated miRNAs (miR-375, miR-125b, miR-146a)" in CSF (see above). Strikingly, heatmap analysis showed several classes of miRNAs that derived from the 28 studies (Figure 4). We used three GO analysis tools - DAVID, FunRich and String - to assess biological functions of coordinately regulated miRNAs with common targets and these data were visualized using bar graphs (Figure 5). Remarkably, our assessment showed that the putative functions of up-regulated miRNAs primarily target the nucleus and down-regulated miRNAs primarily target transcription, DNA-templated (Figure 5). These results of the analysis suggest that up- and down-regulated miRNAs biologically target two diverse molecular mechanisms to affect AD.

\section{Discussion}

More and more studies have demonstrated that miRNAs play a crucial role as candidate biomarkers of AD for early diagnosis or new drug targets, which take part in posttranscriptional regulation of expression of various genes. The benefit of biomarkers is to detect the pathology, especially since it is not present in the preclinical phase early. A miRNA may have hundreds of different mRNA targets; at the same time a target might be regulated by a combination of multiple miRNAs [27]. In $A D$ dysregulated miRNAs have been identified to be involved in amyloidogenesis, inflammation, tau phosphorylation, apoptosis, synaptogenesis, neurotrophism, neurons degradation, innate immune signaling, NF- $\kappa B$ signaling, and activation of cell cycle entry.

The name "Alzheimer's disease" pertains to the underlying pathological features: neuronal atrophy, synapse loss and the progressive accumulation of senile plaques. These plaques are composed of various amyloid $\beta(A \beta)$ peptides, including amyloid precursor protein ( $A \beta 40$ and $A \beta$ 42) and intracellular neurofibrillary tangles (NFTs) 
A

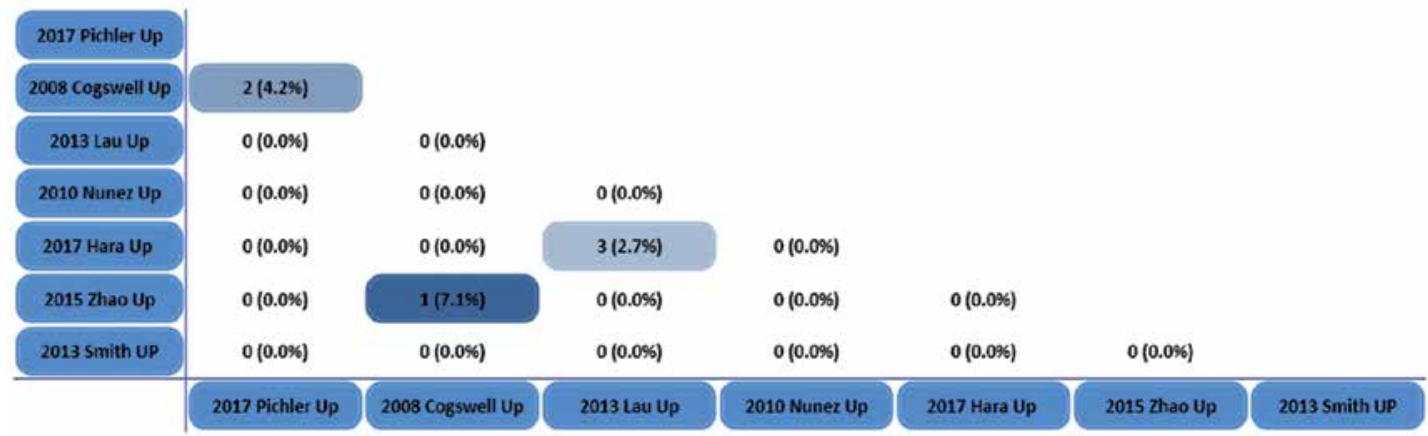

B

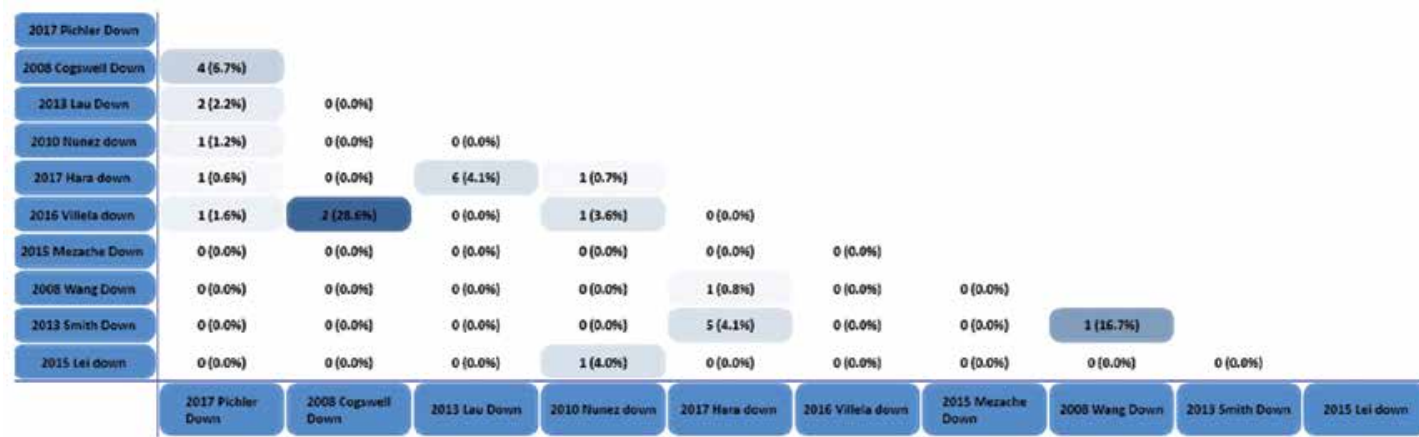

C

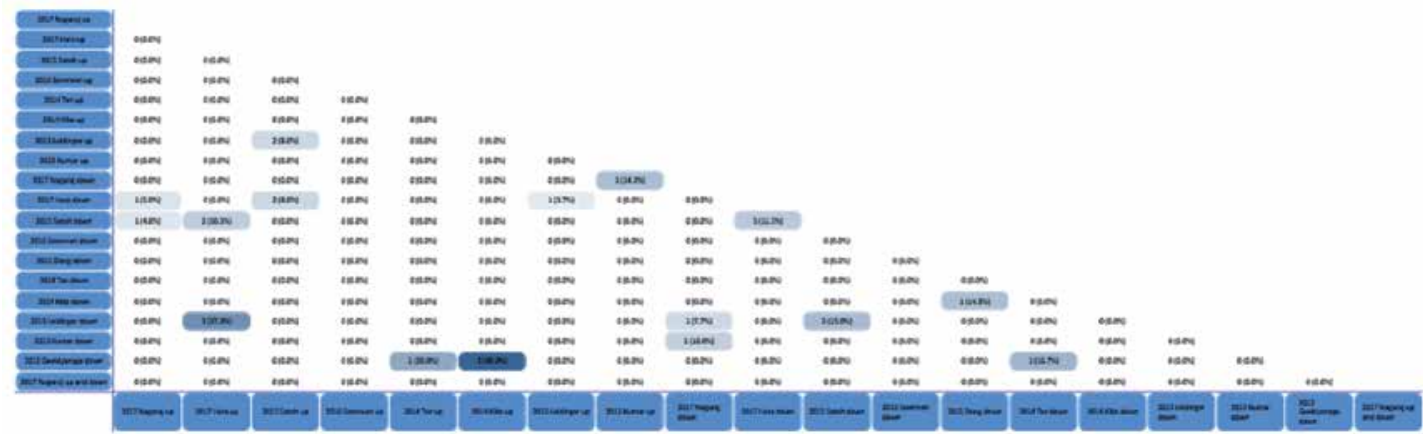

D

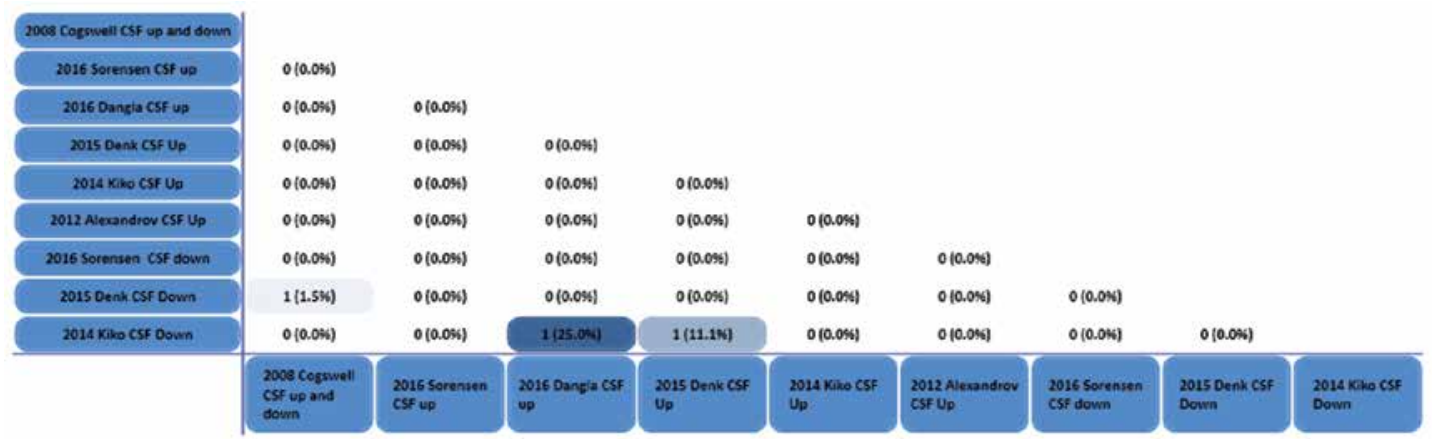

Figure 2. Tabular Venn diagram analysis. Cross-tables show the number and percentage of miRNAs that are in common among studies examining miRNA expression by microarray analysis. A - up-regulation in cortex; B - down-regulation in cortex; C - dysregulation in blood; D - dysregulation in CSF. The number of miRNAs in common to each of the groups is rather. No, that means the common miRNAs in different studies and it reveals that none of the studies reported the same up- and down-regulated miRNAs from a the same class 


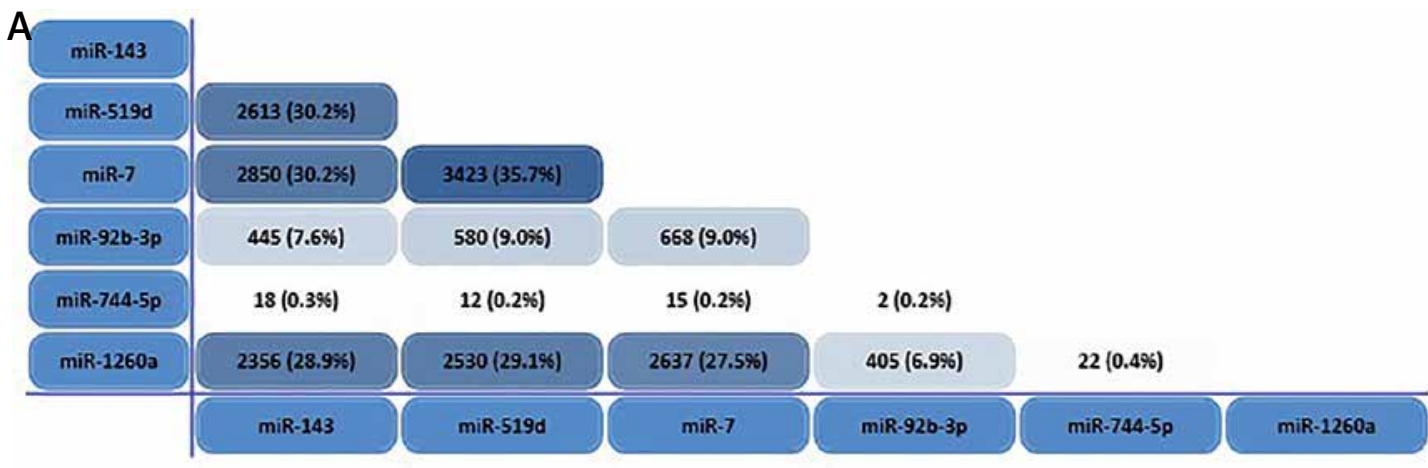

B

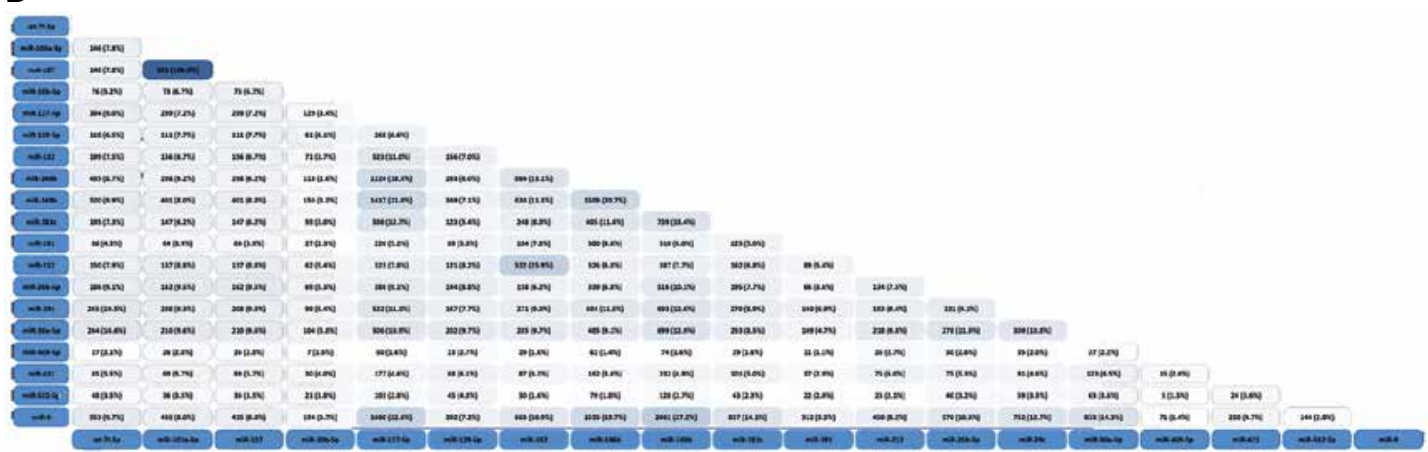

Figure 3. Tabular Venn diagram analysis. The cross-table in panel A is based on up- or down-regulated miRNAs that are identified in two or more studies depicted in Figures $2 \mathrm{~A}$ and $\mathrm{B}$, and shows the similarities in predicted target genes of up-regulated miRNAs. Panel B shows the same as Panel A for putative target genes of down-regulated miRNAs identified in Figures $2 \mathrm{~A}$ and $\mathrm{B}$ as having been observed in two or more studies

A
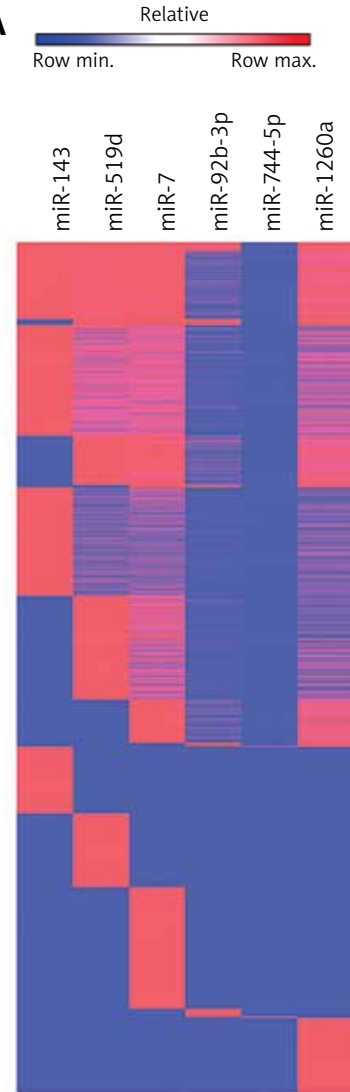

B

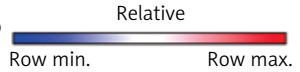

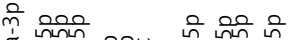

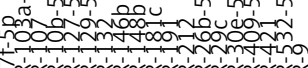
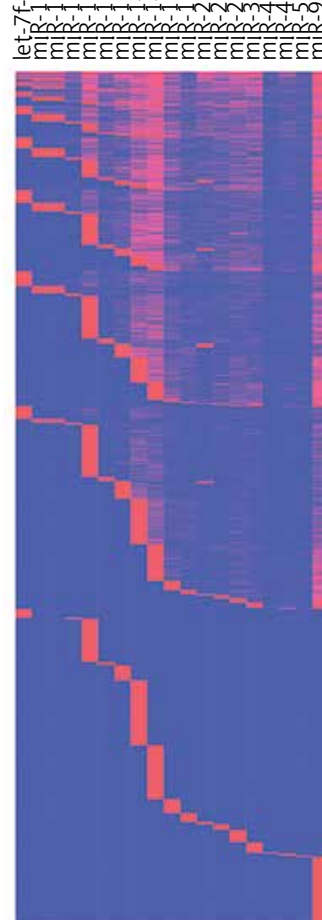

C
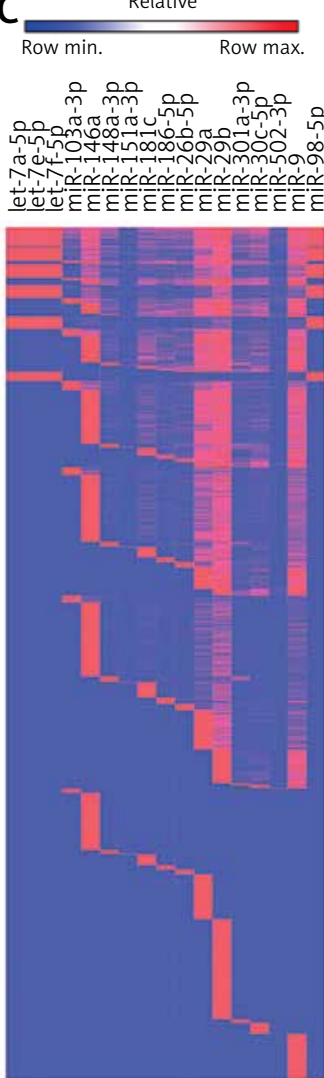
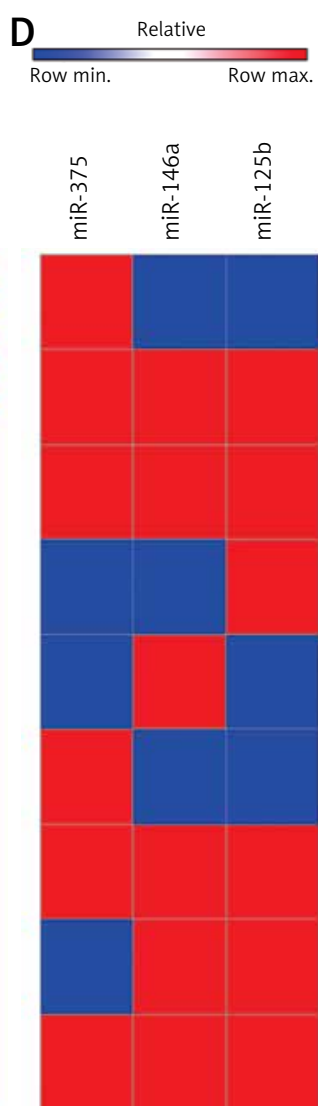

Figure 4. Identification of common miRNAs and their targets in AD. The figure shows heatmap analyses of the relatedness between studies examining miRNA expression ( $\mathbf{A}$ - up-regulation in cortex; $\mathbf{B}$ - down-regulation in cortex; C - dysregulation in blood; D - dysregulation in CSF), miRNAs identified as differentially expressed in AD, and the predicted gene targets of these miRNAs 
A

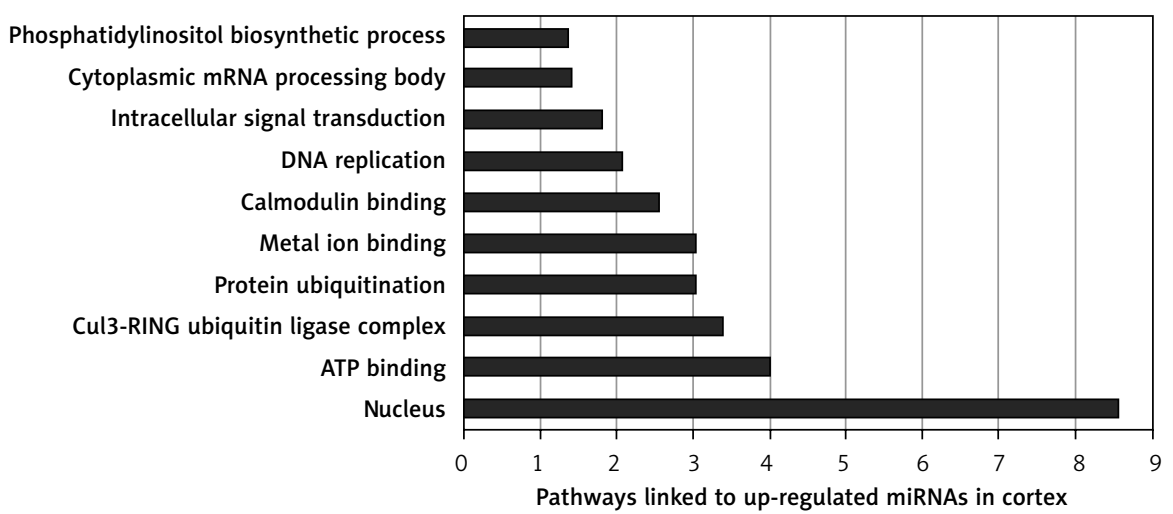

B

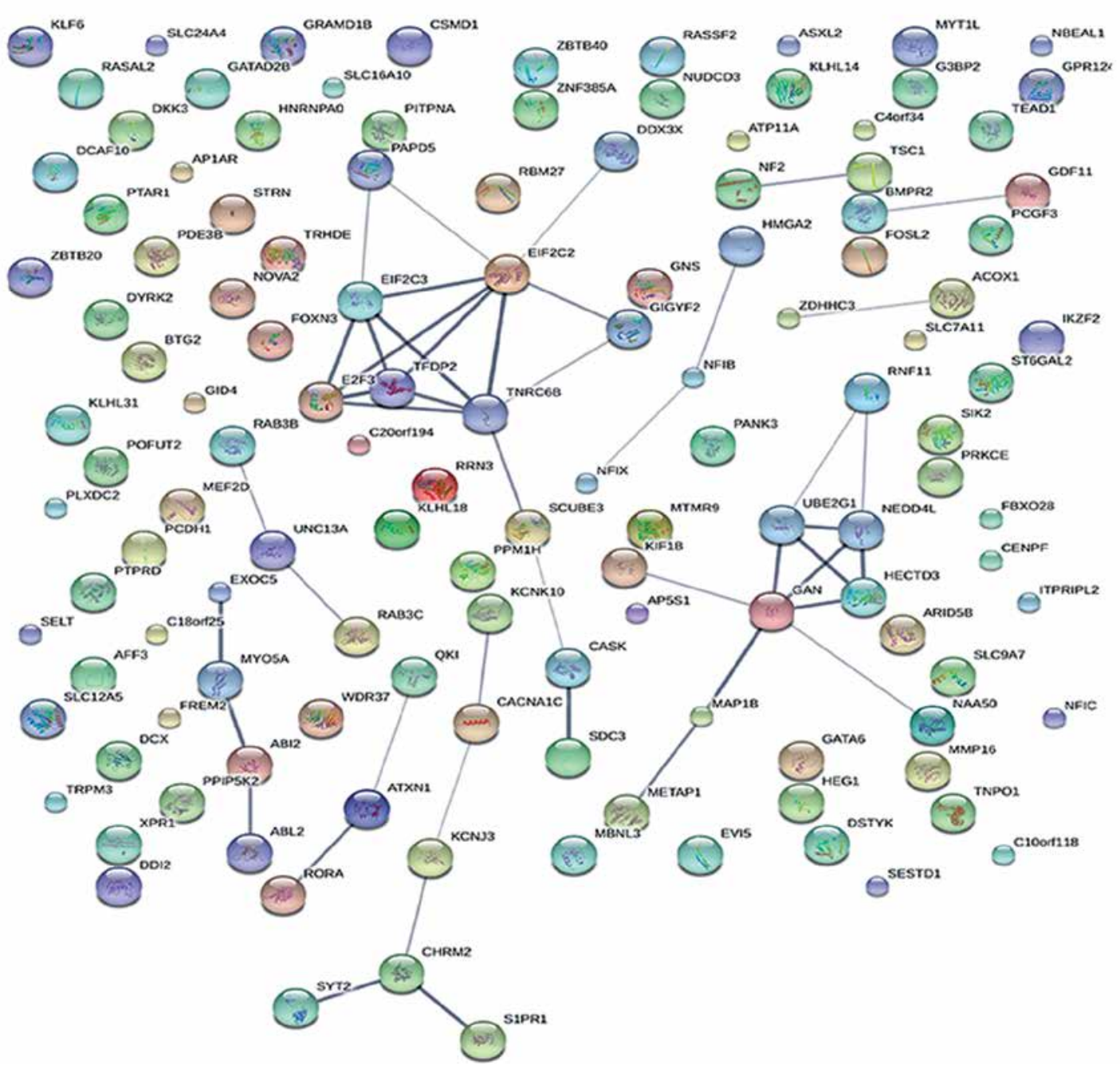

Figure 5. Common targets of $A D$ related miRNAs are linked to distinct biological processes. Different classes of common miRNAs associated with AD collectively target distinct gene networks. A - the top 10 functions linked to up-regulated miRNAs. B - the networks of common genes linked to up-regulated miRNAs

(hyperphosphorylated tau protein) [28]. Cerebrovascular deregulation is seen as a contributor to AD [29], providing a better understanding of the physiological mechanisms of $A D$ in dementia, which helps us to focus on the treatment of neurovascular repair. MiRNAs related to cerebrovas- cular deregulation may play an important role in AD, which helps us collect comprehensive data to assess miRNAs in AD.

The pathological features are, along with synaptic transmission, synaptic plasticity, learning, and memory; neuronal survival and apoptosis; 
C

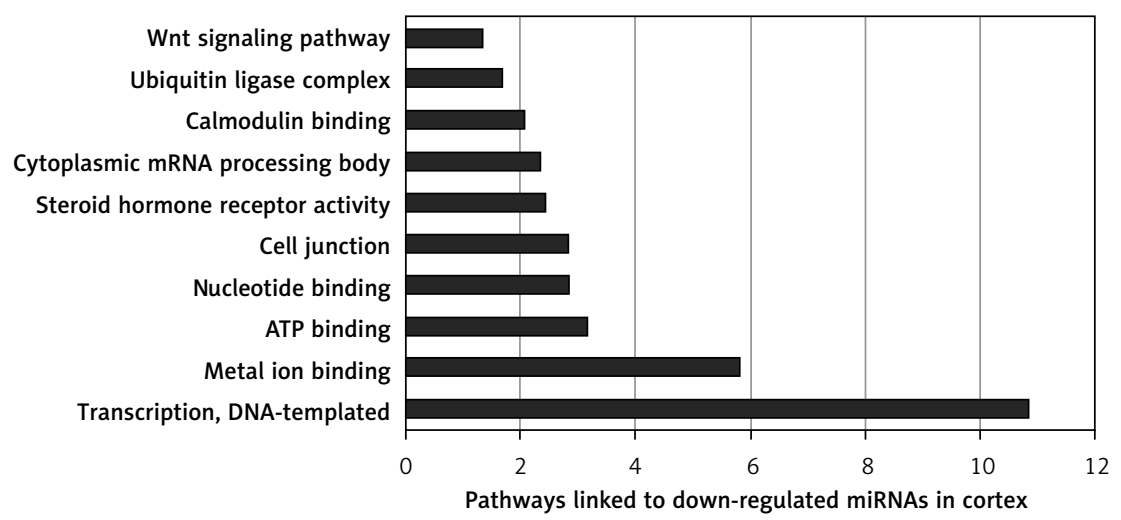

D

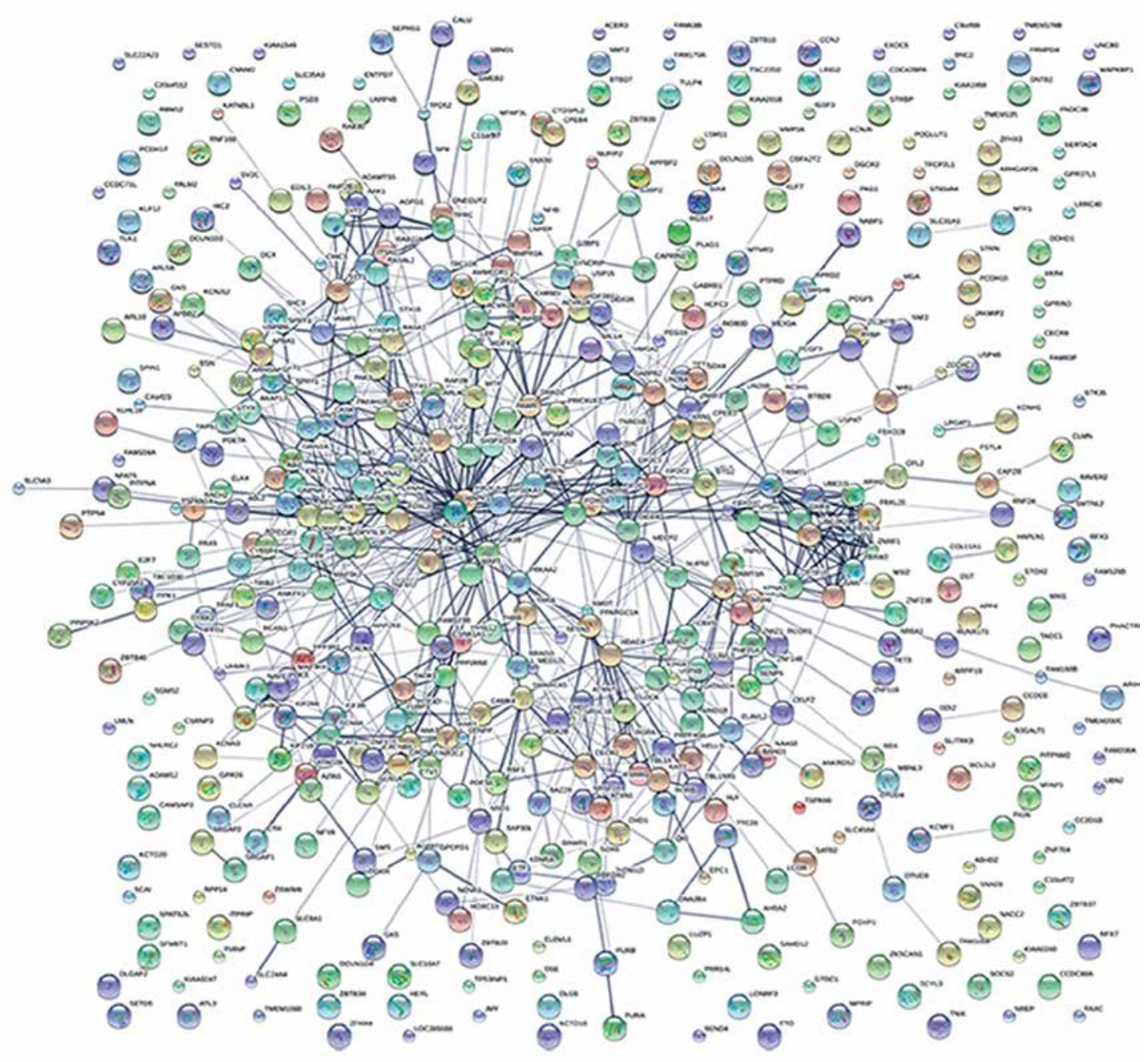

Figure 5. Cont. $\mathbf{C}$ - the top 10 functions linked to down-regulated miRNAs. $\mathbf{D}$ - the networks of common genes linked to down-regulated miRNAs

adult neurogenesis which continues in the adult hippocampus; regulation of neural processes and structural plasticity; inflammation; and amyloid $\beta(A \beta)$ production and toxicity. Increasingly, numerous miRNAs have been found to play crucial roles in regulating $A \beta$ production in the process of $A D[30]$.
$A D$ markers mostly mentioned are tau and $A \beta$, which are used to identify and understand the mechanisms involved in the etiopathogenesis of AD. Amyloidogenesis reflects the pathological features of $A D$ and plays a remarkable role in $A D$ progression. Many studies have indicated that dysregulation of miRNAs has been linked with 
tau and $A \beta$ pathogenic functions in AD. Until now, many studies have implicated miRNAs in amyloidogenesis, especially as a central factor in $A D$, such as miR-7 [9, 22], miR-9 [9, 15], miR-26b [14, 30], miR-34a [31], miR-98-5p [32], miR-106 [33], miR-128 [34], miR-132 [35], miR-144 [36], miR146a [37], miR-153 [38], miR-181 [39], miR-211$5 p$ [40] and miR-339-5p [41]. And the prefibrillar form of soluble $A \beta$ also impairs synaptic function and is the major cause of cognitive defects associated with AD. For instance, some studies reported that miRNAs including miR-125b [22, 42, 43], miR134 [44], and miR-384 [45] may dysregulate $A \beta$ to cause synaptic dysfunction.

Neuronal differentiation is also a part of $A D$ pathology including neuronal survival and apoptosis, morphogenesis, adult neurogenesis, regulation of neural processes and structural plasticity. Several miRNAs have been identified as related to neuronal differentiation including miR-26b [14, 30], miR-29 [46-49], miR-34b/c [50, 51], miR-107 [52, 53], miR-124 [54], miR-128 [34], miR-137 [55], miR-193b [56], miR-210 [57], miR-211-5p [40] and miR-384 [45].

Microglia are a kind of immunocompetent cells in the central nervous system (CNS), which accumulates at amyloid $\beta$ plaques in $A D$ as activated microglia. Several miRNAs may preferentially block many cellular anti-inflammatory proteins' translation that could drive a pro-inflammatory response [58]. Key pro-inflammatory (miR-155 [19, 22], miR-27b [12], miR-326 [14]), anti-inflammatory (miR-9 $[9,15]$, miRNA-125b [43], miR124 [5, 12, 25], miR-132 [35], miR-146a [19, 37], miR-21 [12], miR-223 [12]), and mixed immunomodulatory (let-7 family [8, 12, 14, 16, 24, 59, 60]) miRNAs regulate neuroinflammation in $A D$. In the absence of a pro-inflammatory response, inflammasome-mediated collagen synthesis could not be induced. MiRNA-146a was reported [19] to be related inflammation in $A D$ as an NF- $\mathrm{B} B$-regulated pro-inflammatory miRNA which targets the 3'-UTR of complement factor $\mathrm{H}(\mathrm{CFH})$ in human monocytes.

Bioinformatics analysis of quantitative expression data was performed, identifying a variety of miRNAs as up- or down- regulated in AD cortex, blood and CSF compared to age-matched controls. Increasingly many studies focus on miRNA dysregulation not only in $A D$ brain but also in $A D$ blood and CSF and have yielded better convergence of the database to improve the detection methods for miRNAs. In spite of inconsistent upor down-regulated miRNAs, it is a research trend to find the key biomarkers in the preclinical phase early.

Bioinformatic analysis was used to compare gene ontology terms and to assess whether the common targets of representative coordinately regulated miRNAs play a key role in larger biological programs and regulatory networks. We separately listed the top 10 functions of two groups of miRNAs from our database (Figure 5) to show whether these miRNAs have the same predicted targets. Then, we resorted to Target Scan to find and tabulate potential target mRNAs. This analysis revealed that six common miRNAs that are reported as up-regulated in $A D$ cortex (i.e., miR143, miR-519d, miR-7, miR-92b-3p, miR-744-5p, miR-1260a) in these studies have many targets in common (Figure 4). Similarly, twenty common down-regulated miRNAs observed in these studies (i.e., let-7f-5p, miR-103a-3p, miR-107, miR-10b-5p, miR-127-5p, miR-129-5p, miR-132, miR-146b, miR148b, miR-181c, miR-191, miR-212, miR-26b-5p, miR-29c, miR-30e-5p, miR-409-5p, miR-421, miR496, miR-532-5p, miR-9) also have a shared set of targets (Figure 4). But in AD blood and CSF there are not consistent up- or down-regulated miRNAs in different studies. We listed the common miRNAs related to $A D$ (let-7a-5p, let-7e-5p, let-7f-5p, miR103a-3p, miR-142-3p, miR-146a, miR-148a-3p, miR-151a-3p, miR-181c, miR-186-5p, miR-26b-5p, miR-29a, miR-29b, miR-301a-3p, miR-30c-5p, miR502-3p, miR-9, miR-98-5p in blood and miR-375, miR-125b, miR-146a) but the results of dysregulation are not consistent (Figure 4).

Divergence of miRNA dysregulation may be caused by several factors: 1) miRNA regulation altered in different stages of $A D$ is different. If each study only compared the same phase of AD with healthy controls, there should be the same results, but these studies compared all phases of AD with healthy controls, leading to the opposite results. The same miRNA can target different genes to cause the pathology of AD. In turn, the impacts of genes on miRNAs cause different miRNA regulation. Onset of miRNA functional deficits in the different stages of AD may be different. 2) Different miRNA detection may produce different results because of sensitivity and specificity. 3) The insufficient samples led to weakening of statistical characteristics and resulted in different data or unclear mechanisms of AD. So the essential question remained unaddressed: whether these miRNAs are upregulated or downregulated in different tissue of $A D$ and whether specific or early perturbations of miRNA regulation cause the link to relevant mechanisms of $A D$ pathology or only nonspecific epiphenomena of neurodegeneration. The further study must overcome the challenges posed by studies with limited patient sample sizes. Cause and effect are difficult to establish in our assessment that many miRNAs were misregulated and it is suggestive that dysregulation were not merely consequences of pathology. Challenges for 
further research are posed by studies with different tissues and limited patient sample sizes. Taken together, both up- and down-regulated miRNAs in $A D$ different specimens seem to coordinately suppress a common set of genes. We used GO terms to get larger biological programs and regulatory networks from our obtained data about representative coordinately regulated miRNAs' common targets in cortex. This analysis resulted in the striking finding that shared miRNAs detected in common up-regulated miRNAs primarily target the nucleus and common down-regulated miRNAs primarily target transcription, DNA-templated. In the nucleus, miRNAs regulate gene expression by binding to the targeted promoter sequences and affect either the transcriptional gene silencing (TGS) or transcriptional gene activation (TGA) [61]. Distinct common sets of mRNAs link the respective targets and broader biological programs, which may indicate that AD may be guided by effects of proteins required for gene expression.

Most of the mRNAs targeted by miRNAs are transcription factors (TFs), which focus on regulating plant growth and development [62]. The observation is consistent with a relatively simple molecular framework for miRNA-mediated nerve degeneration. Dysregulated miRNAs play an important role in progression of $A D$, regardless as to whether miRNAs are up-regulated or down-regulated. Modulation of any processing by miRNAs can achieve a 'double-negative principle. For example, up-regulated miR-143, miR-519d, miR-7, miR-92b-3p, miR-744-5p, miR-1260a could upregulate TFs or other positive proteins and lower expression of negative proteins, and then lead to high gene expression, which may be protective of CNS. Furthermore, down-regulated let-7f-5p, miR-103a-3p, miR-107, miR-10b-5p, miR-127-5p, miR-129-5p, miR-132, miR-146b, miR-148b, miR181c, miR-191, miR-212, miR-26b-5p, miR-29c, miR-30e-5p, miR-409-5p, miR-421, miR-496, miR532-5p, miR-9 could lower expression of positive TFs or raise expression of negative TFs, and then lead to CNS degenerative gene overexpression or protective gene under-expression. But the molecular mechanisms are still mostly unclear. We speculated that perturbations in miRNA-TF regulatory networks during successive stages of AD may be caused by broad ranging pleiotropic effects.

$A D$ activity remains largely enigmatic because it is multi-factorial, with a range of processes compromised. However, implicated miRNA pathways, which control transcription, cell junction and ATP binding kinase-related signaling pathways, have been increasingly identified. In addition they can lead to amyloidogenesis, inflammation, tau phosphorylation, apoptosis, synaptogenesis, neurotrophism, neuron degradation, and activation of cell cycle entry. According to screening of AD-related miRNAs, we can identify the preclinical phase early, which provides an important time window for therapeutic intervention. We can better understand the unknown mechanisms of progres sive $A D$ pathology. It is unclear which miRNAs are primarily susceptible to $A D$ through $A D$-miRNAs screening, but rather it indicates $A D$ activity more easily than would otherwise have been the case. There are pronounced clinical implications for assessing the risk miRNAs related to AD. Our study comprehensively reviewed the miRNAs' dysregulation in different tissues of AD, and it can be used to delay disease progression. Recently more and more studies have tried to identify possible miRNA therapeutic targets. However, it is a long way to use the predicted therapeutic benefits of targeted treatments in clinical practice without clinical trials, which requires the safety and efficacy of targeted drugs.

There are advantages and limitations of this study. Although we conducted a comprehensive search through multiple databases for selection and appraisal of candidate AD miRNA biomarkers by independent pairs of reviewers, including several hypotheses for the role of miRNAs in AD, our study suffered several important limitations: 1) Divergence of miRNA dysregulation may be caused by the different phases of AD. If each study only compared the same phase of AD with healthy controls, there should be the same results, but these studies compared all phases of AD with healthy controls, leading to the opposite results. It may be because the insufficient samples led to weakening of statistical characteristics and resulted in different data. So it is better to collect larger samples to assess the regulation of miRNAs in AD pathogenesis. There may be publication bias because of inadvertently omitting unpublished studies with negative results. 2) There are other unknown biological processes caused by miRNA dysregulation in AD. The further study will be designed well to assess the roles of miRNAs in the different phases of $A D$ and it is better to evaluate the dysregulation of microRNAs related to $A D$ to reveal the underlying complex mechanisms. Therefore, in the future we need to renew the miRNA-AD database.

In conclusion, we provided a comprehensive genome scale description of disease-altered expression of miRNAs in AD brain, blood and CSF. Through our search and assessment of miRNA targets, we identified what miRNAs may correlate with useful biomarkers of $A D$ and be referred to novel pathways contributing to $A D$ pathogenesis. In addition to dysregulation of miRNAs in $A D$ brain, there are quantitative changes in miRNA expression in $A D$ blood and CSF that provide initial hope to provide accessible biomarkers to aid diag- 
nosis of $A D$. In the future, it is advisable to focus on the relationship between the miRNA changes in blood and CSF and AD-specific signaling molecules and aspects of these changes specific to AD.

Even though hundreds of miRNAs have been identified as dysregulated, it is unknown which miRNAs are the primary biomarkers. Bioinformatics analysis of dysregulated miRNAs in $A D$ reveals a relationship in miRNA signatures of different tissues. We identified that miRNAs target TFs to directly control gene expression, which is a unique miRNA signature, while the other studies indicate different miRNAs that control nucleus functions. It is crucially important to diagnose AD combined with dysregulated miRNA profiles and other standard diagnostic tools at the preclinical stages. Additionally, miRNA profiling from brain, blood or CSF samples for future research will achieve the same AD diagnosis of the same patient.

\section{Acknowledgments}

This work was supported by Chuangxin Fund of Navy General Hospital (201604). The funders had no role in the study design, data collection and analysis, decision to publish, or preparation of the manuscript.

\section{Conflict of interest}

The authors declare no conflict of interest.

\section{References}

1. Wierzejska R. Can coffee consumption lower the risk of Alzheimer's disease and Parkinson's disease? A literature review. Arch Med Sci 2017; 13: 507-14.

2. Bathina S, Das UN. Brain-derived neurotrophic factor and its clinical implications. Arch Med Sci 2015; 11: 1164-78.

3. Esquela-Kerscher A, Slack FJ. Oncomirs - microRNAs with a role in cancer. Nat Rev Cancer 2006; 6: 259-69.

4. Cogswell JP, Ward J, Taylor IA, et al. Identification of miRNA changes in Alzheimer's disease brain and CSF yields putative biomarkers and insights into disease pathways. J Alzheimer's Dis 2008; 14: 27-41.

5. Lukiw WJ. Micro-RNA speciation in fetal, adult and Alzheimer's disease hippocampus. Neuroreport 2007; 18: 297-300.

6. Sethi P, Lukiw WJ. Micro-RNA abundance and stability in human brain: specific alterations in Alzheimer's disease temporal lobe neocortex. Neurosci Lett 2009; 459: 1004.

7. Hill JM, Pogue Al, Lukiw WJ. Pathogenic microRNAs common to brain and retinal degeneration; recent observations in Alzheimer's disease and age-related macular degeneration. Front Neurol 2015; 6: 232.

8. Satoh J, Kino Y, Niida S. MicroRNA-Seq data analysis pipeline to identify blood biomarkers for Alzheimer's disease from public data. Biomarker Insights 2015; 10: 21-31.

9. Pichler S, Gu W, Hartl D, et al. The miRNome of Alzheimer's disease: consistent downregulation of the miR-
132/212 cluster. Neurobiol Aging 2017; 50: 167.e161167.e110.

10. Nagaraj S, Laskowska-Kaszub K, Debski KJ, et al. Profile of 6 microRNA in blood plasma distinguish early stage Alzheimer's disease patients from non-demented subjects. Oncotarget 2017; 8: 16122-43.

11. Lau P, Bossers K, Janky R, et al. Alteration of the microRNA network during the progression of Alzheimer's disease. EMBO Mol Med 2013; 5: 1613-34.

12. Wang WX, Huang Q, Hu Y, Stromberg AJ, Nelson PT. Patterns of microRNA expression in normal and early Alzheimer's disease human temporal cortex: white matter versus gray matter. Acta Neuropathol 2011; 121: 193-205.

13. Panic N, Leoncini E, de Belvis G, Ricciardi W, Boccia S. Evaluation of the endorsement of the preferred reporting items for systematic reviews and meta-analysis (PRISMA) statement on the quality of published systematic review and meta-analyses. PLoS One 2013; 8: e83138.

14. Hara N, Kikuchi M, Miyashita A, et al. Serum microRNA miR-501-3p as a potential biomarker related to the progression of Alzheimer's disease. Acta Neuropathol Commun 2017; 5: 10.

15. Villela D, Ramalho RF, Silva AR, et al. Differential DNA methylation of microRNA genes in temporal cortex from Alzheimer's disease individuals. Neural Plast 2016; 2016: 2584940.

16. Sorensen SS, Nygaard AB, Christensen T. miRNA expression profiles in cerebrospinal fluid and blood of patients with Alzheimer's disease and other types of dementia - an exploratory study. Transl Neurodegener 2016; 5: 6.

17. Dangla-Valls A, Molinuevo JL, Altirriba J, et al. CSF microRNA profiling in Alzheimer's disease: a screening and validation study. Mol Neurobiol 2017; 54: 6647-54.

18. Tan L, Yu JT, Tan MS, et al. Genome-wide serum microRNA expression profiling identifies serum biomarkers for Alzheimer's disease. J Alzheimer's Dis 2014; 40: 1017-27.

19. Lukiw WJ, Surjyadipta B, Dua P, Alexandrov PN. Common micro RNAs (miRNAs) target complement factor $\mathrm{H}$ (CFH) regulation in Alzheimer's disease (AD) and in age-related macular degeneration (AMD). Int J Biochem Mol Biol 2012; 3: 105-16.

20. Schonrock N, Matamales M, Ittner LM, Gotz J. MicroRNA networks surrounding APP and amyloid-beta metabolism--implications for Alzheimer's disease. Exp Neurol 2012; 235: 447-54.

21. Krichevsky AM, King KS, Donahue CP, Khrapko K, Kosik KS. A microRNA array reveals extensive regulation of microRNAs during brain development. RNA 2003; 9 : 1274-81.

22. Zhao Y, Bhattacharjee S, Dua P, Alexandrov PN, Lukiw WJ. MicroRNA-based biomarkers and the diagnosis of Alzheimer's disease. Front Neurol 2015; 6: 162.

23. Mezache L, Mikhail M, Garofalo M, Nuovo GJ. Reduced miR-512 and the elevated expression of its targets CFLIP and MCL1 localize to neurons with hyperphosphorylated tau protein in Alzheimer disease. Appl Immunohistochem Mol Morphol 2015; 23: 615-23.

24. Leidinger P, Backes C, Deutscher S, et al. A blood based 12-miRNA signature of Alzheimer disease patients. Genome Biol 2013; 14: R78.

25. Nunez-Iglesias J, Liu CC, Morgan TE, Finch CE, Zhou XJ. Joint genome-wide profiling of miRNA and mRNA expression in Alzheimer's disease cortex reveals altered miRNA regulation. PLoS One 2010; 5: e8898.

26. Kiko T, Nakagawa K, Tsuduki T, Furukawa K, Arai H, Miyazawa T. MicroRNAs in plasma and cerebrospinal fluid 
as potential markers for Alzheimer's disease. J Alzheimer's Dis 2014; 39: 253-9.

27. Friedman RC, Farh KK, Burge CB, Bartel DP. Most mammalian mRNAs are conserved targets of microRNAs. Genome Res 2009; 19: 92-105.

28. Karran E, Mercken M, De Strooper B. The amyloid cascade hypothesis for Alzheimer's disease: an appraisal for the development of therapeutics. Nature Rev Drug Discov 2011; 10: 698-712.

29. Schaller BJ. Strategies for molecular imaging dementia and neurodegenerative diseases. Neuropsychiatr Dis Treatment 2008; 4: 585-612.

30. Absalon S, Kochanek DM, Raghavan V, Krichevsky AM. MiR-26b, upregulated in Alzheimer's disease, activates cell cycle entry, tau-phosphorylation, and apoptosis in postmitotic neurons. J Neurosci 2013; 33: 14645-59.

31. Sarkar S, Jun S, Rellick S, Quintana DD, Cavendish JZ, Simpkins JW. Expression of microRNA-34a in Alzheimer's disease brain targets genes linked to synaptic plasticity, energy metabolism, and resting state network activity. Brain Res 2016; 1646: 139-51.

32. Li Q, Li X, Wang L, Zhang Y, Chen L. miR-98-5p acts as a target for Alzheimer's disease by regulating abeta production through modulating SNX6 expression. J Mol Neurosci 2016; 60: 413-20.

33. Kim J, Yoon H, Ramirez CM, et al. MiR-106b impairs cholesterol efflux and increases Abeta levels by repressing ABCA1 expression. Exp Neurol 2012; 235: 476-83.

34. Tiribuzi R, Crispoltoni L, Porcellati S, et al. miR128 up-regulation correlates with impaired amyloid beta(1-42) degradation in monocytes from patients with sporadic Alzheimer's disease. Neurobiol Aging 2014; 35: 345-56.

35. Salta E, De Strooper B. microRNA-132: a key noncoding RNA operating in the cellular phase of Alzheimer's disease. FASEB J 2017; 31: 424-33.

36. Cheng C, Li W, Zhang Z, et al. MicroRNA-144 is regulated by activator protein-1 (AP-1) and decreases expression of Alzheimer disease-related a disintegrin and metalloprotease 10 (ADAM10). J Biol Chem 2013; 288: 13748-61.

37. Cui L, Li Y, Ma G, et al. A functional polymorphism in the promoter region of microRNA-146a is associated with the risk of Alzheimer disease and the rate of cognitive decline in patients. PLoS One 2014; 9: e89019.

38. Liang $\mathrm{C}$, Zhu $\mathrm{H}, \mathrm{Xu}$ Y, et al. MicroRNA-153 negatively regulates the expression of amyloid precursor protein and amyloid precursor-like protein 2. Brain Res 2012; 1455: 103-13.

39. Schonrock N, Humphreys DT, Preiss T, Gotz J. Target gene repression mediated by miRNAs miR-181c and miR-9 both of which are down-regulated by amyloid-beta. J Mol Neurosci 2012; 46: 324-35.

40. Fan C, Wu Q, Ye X, et al. Role of miR-211 in neuronal differentiation and viability: implications to pathogenesis of Alzheimer's disease. Front Aging Neurosci 2016; 8: 166.

41. Long JM, Ray B, Lahiri DK. MicroRNA-339-5p down-regulates protein expression of beta-site amyloid precursor protein-cleaving enzyme 1 (BACE1) in human primary brain cultures and is reduced in brain tissue specimens of Alzheimer disease subjects. J Biol Chem 2014; 289 . 5184-98.

42. Tan L, Yu JT, Liu QY, et al. Circulating miR-125b as a biomarker of Alzheimer's disease. J Neurol Sci 2014; 336: 52-56.

43. Banzhaf-Strathmann J, Benito E, May S, et al. MicroRNA-125b induces tau hyperphosphorylation and cognitive deficits in Alzheimer's disease. EMBO J 2014; 33: 1667-80.
44. Riancho J, Vazquez-Higuera JL, Pozueta A, et al. MicroRNA profile in patients with Alzheimer's disease: analysis of miR-9-5p and miR-598 in raw and exosome enriched cerebrospinal fluid samples. J Alzheimer's Dis 2017; 57: 483-91.

45. Liu CG, Wang JL, Li L, Wang PC. MicroRNA-384 regulates both amyloid precursor protein and beta-secretase expression and is a potential biomarker for Alzheimer's disease. Int J Mol Med 2014; 34: 160-6.

46. Pereira PA, Tomas JF, Queiroz JA, Figueiras AR, Sousa F. Recombinant pre-miR-29b for Alzheimer s disease therapeutics. Sci Rep 2016; 6: 19946

47. Villa C, Ridolfi E, Fenoglio C, et al. Expression of the transcription factor Sp1 and its regulatory hsa-miR-29b in peripheral blood mononuclear cells from patients with Alzheimer's disease. J Alzheimer's Dis 2013; 35: 487-94.

48. Lei X, Lei L, Zhang Z, Zhang Z, Cheng Y. Downregulated miR-29c correlates with increased BACE1 expression in sporadic Alzheimer's disease. Int J Clin Exp Pathol 2015; 8: 1565-74.

49. Geekiyanage H, Jicha GA, Nelson PT, Chan C. Blood serum miRNA: non-invasive biomarkers for Alzheimer's disease. Exp Neurol 2012; 235: 491-6.

50. Zovoilis A, Agbemenyah HY, Agis-Balboa RC, et al. microRNA-34C is a novel target to treat dementias. EMBO 2011; 30: 4299-308.

51. Bhatnagar S, Chertkow H, Schipper HM, et al. Increased microRNA-34c abundance in Alzheimer's disease circulating blood plasma. Front Mol Neurosci 2014; 7: 2.

52. Jiao Y, Kong L, Yao Y, et al. Osthole decreases beta amyloid levels through up-regulation of miR-107 in Alzheimer's disease. Neuropharmacology 2016; 108: 332-44.

53. Goodall EF, Heath PR, Bandmann O, Kirby J, Shaw PJ. Neuronal dark matter: the emerging role of microRNAs in neurodegeneration. Front Cell Neurosci 2013; 7: 178.

54. Fang $M$, Wang J, Zhang X, et al. The miR-124 regulates the expression of BACE1/beta-secretase correlated with cell death in Alzheimer's disease. Toxicol Lett 2012; 209: 94-105.

55. Geekiyanage H, Chan C. MicroRNA-137/181c regulates serine palmitoyltransferase and in turn amyloid beta, novel targets in sporadic Alzheimer's disease. J Neurosci 2011; 31: 14820-30

56. Liu CG, Song J, Zhang YQ, Wang PC. MicroRNA-193b is a regulator of amyloid precursor protein in the blood and cerebrospinal fluid derived exosomal microRNA-193b is a biomarker of Alzheimer's disease. Mol Med Rep 2014; 10: 2395-400.

57. Zhu Y, Li C, Sun A, Wang Y, Zhou S. Quantification of microRNA-210 in the cerebrospinal fluid and serum: implications for Alzheimer's disease. Exp Ther Med 2015; 9: 1013-7.

58. Ghribi O. NF-kappaB - a friend, a foe, or a bystander - in the neurodegenerative cascade and pathogenesis of Alzheimer's disease. CNS Neurol Disord Drug Targets 2017; 16: 1050-65.

59. Smith-Vikos T, Slack FJ. MicroRNAs circulate around Alzheimer's disease. Genome Biol 2013; 14: 125.

60. Kumar P, Dezso Z, MacKenzie C, et al. Circulating miRNA biomarkers for Alzheimer's disease. PLoS One 2013; 8 : e69807.

61. Kotipalli A, Gutti R, Mitra CK. Dynamics of miRNA biogenesis and nuclear transport. J Integr Bioinform 2016 13: 305 .

62. Samad AFA, Sajad M, Nazaruddin N, et al. MicroRNA and transcription factor: key players in plant regulatory network. Front Plant Sci 2017; 8: 565. 